\title{
A note on homogeneous Sobolev spaces of fractional order
}

\author{
${\text { Lorenzo } \text { Brasco }^{1} \text { (D) } \cdot \text { Ariel Salort }}^{2}$
}

Received: 18 May 2018 / Accepted: 15 December 2018 / Published online: 3 January 2019

(c) Fondazione Annali di Matematica Pura ed Applicata and Springer-Verlag GmbH Germany, part of Springer Nature 2019

\begin{abstract}
We consider a homogeneous fractional Sobolev space obtained by completion of the space of smooth test functions, with respect to a Sobolev-Slobodeckii norm. We compare it to the fractional Sobolev space obtained by the $K$-method in real interpolation theory. We show that the two spaces do not always coincide and give some sufficient conditions on the open sets for this to happen. We also highlight some unnatural behaviors of the interpolation space. The treatment is as self-contained as possible.
\end{abstract}

Keywords Nonlocal operators · Fractional Sobolev spaces · Real interpolation · Poincaré inequality

Mathematics Subject Classification 46E35 - 46B70

\section{Contents}

1 Introduction . . . . . . . . . . . . . . . . . . . . . . . . . 1296

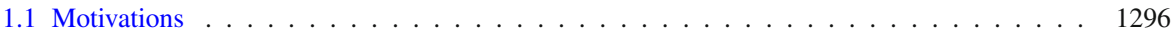

1.2 Aims . . . . . . . . . . . . . . . . . . . . . . . . . . . 1297

1.3 Results . . . . . . . . . . . . . . . . . . . . . . . . . . . . . . 1298

1.4 Plan of the paper . . . . . . . . . . . . . . . . . . . . . . . . . 1300

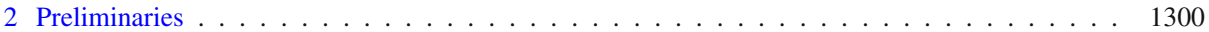

2.1 Basic notation . . . . . . . . . . . . . . . . . . . . . . . . 1300

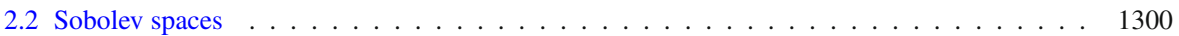

2.3 A homogeneous Sobolev-Slobodeckiĭ space . . . . . . . . . . . . . . . . . . . . . . . . . 1301

2.4 Another space of functions vanishing at the boundary . . . . . . . . . . . . . . . . . . . 1302

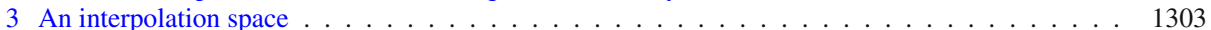

4 Interpolation versus Sobolev-Slobodeckiǔ . . . . . . . . . . . . . . . . . . . . . . 1306

4.1 General sets . . . . . . . . . . . . . . . . . . . . . . . . . . 1306

Lorenzo Brasco

lorenzo.brasco@unife.it

Ariel Salort

asalort@dm.uba.ar

1 Dipartimento di Matematica e Informatica, Università degli Studi di Ferrara, Via Machiavelli 35, 44121 Ferrara, Italy

2 Departamento de Matemática, FCEN Universidad de Buenos Aires and IMAS, CONICET, Buenos Aires, Argentina 
4.2 Convex sets . . . . . . . . . . . . . . . . . . . . . . . . . . . . . . . . . . 1309

4.3 Lipschitz sets and beyond . . . . . . . . . . . . . . . . . . . . . . . . . 1316

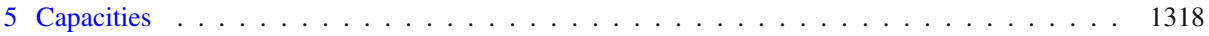

6 Double-sided estimates for Poincaré constants . . . . . . . . . . . . . . . . . . . . . . 1322

Appendix A. An example . . . . . . . . . . . . . . . . . . . . . . . . . . . . . . . . . . . . 1324

Appendix B. One-dimensional Hardy inequality . . . . . . . . . . . . . . . . . . . . . . . 1327

Appendix C. A geometric lemma . . . . . . . . . . . . . . . . . . . . . . . . . . . . 1327

References . . . . . . . . . . . . . . . . . . . . . . . . . . . . 1329

\section{Introduction}

\subsection{Motivations}

In the recent years, there has been a great surge of interest toward Sobolev spaces of fractional order. This is a very classical topic, essentially initiated by the Russian school in the 1950s of the last century, with the main contributions given by Besov, Lizorkin, Nikol'skiŭ, Slobodeckiı̌ and their collaborators. Nowadays, we have a lot of monographies at our disposal on the subject. We just mention the books by Adams [1,2], by Nikol'skiu [25] and by Triebel [3032]. We also refer the reader to [31, Chapter 1] for an historical introduction to the subject.

The reason for this revival lies in the fact that fractional Sobolev spaces seem to play a fundamental role in the study and description of a vast amount of phenomena, involving nonlocal effects. Phenomena of this type have a wide range of applications; we refer to [10] for an overview.

There are many ways to introduce fractional derivatives and, consequently, Sobolev spaces of fractional order. Without any attempt of completeness, let us mention the two approaches which are of interest for our purposes:

- a concrete approach, based on the introduction of explicit norms, which are modeled on the case of Hölder spaces. For example, by using the heuristic

$$
\delta_{h}^{s} u(x):=\frac{u(x+h)-u(x)}{|h|^{s}} \sim \text { "derivative of order } s \text { ", for } x, h \in \mathbb{R}^{N},
$$

a possible choice of norm is

$$
\left(\int\left\|\delta_{h}^{s} u\right\|_{L^{p}}^{p} \frac{\mathrm{d} h}{|h|^{N}}\right)^{\frac{1}{p}}
$$

and more generally

$$
\left(\int\left\|\delta_{h}^{s} u\right\|_{L^{p}}^{q} \frac{\mathrm{d} h}{|h|^{N}}\right)^{\frac{1}{q}}, \quad \text { for } 1 \leq q \leq \infty .
$$

Observe that the integral contains the singular kernel $|h|^{-N}$; thus, functions for which the norm above is finite must be better than just merely $s$-Hölder regular, in an averaged sense;

- an abstract approach, based on the so-called interpolation methods. The foundations of these methods were established at the beginning of the 1960s of the last century, by Calderón, Gagliardo, Krejn, Lions and Petree, among others. A comprehensive treatment of this approach can be found for instance in the books [3,4,29] and references therein. In a nutshell, the idea is to define a scale of "intermediate spaces" between $L^{p}$ and the standard Sobolev space $W^{1, p}$, by means of a general abstract construction. The main 
advantage of this second approach is that many of the properties of the spaces constructed in this way can be extrapolated in a direct way from those of the two "endpoint" spaces $L^{p}$ and $W^{1, p}$.

As mentioned above, actually other approaches are possible: a possibility is to use the Fourier transform. Another particularly elegant approach consists in taking the convolution with a suitable kernel (for example, heat or Poisson kernels are typical choices) and looking at the rate of blowup of selected $L^{p}$ norms with respect to the convolution parameter. However, we will not consider these constructions in the present paper; we refer the reader to [31] for a wide list of definitions of this type.

In spite of the explosion of literature on Calculus of Variations settled in fractional Sobolev spaces of the last years, the abstract approach based on interpolation seems to have been completely neglected or, at least, overlooked. For example, the well-known survey paper [14], which eventually became a standard reference on the field, does not even mention interpolation techniques.

\subsection{Aims}

The main scope of this paper is to revitalize some interest toward interpolation theory in the context of fractional Sobolev spaces. In doing this, we will resist the temptation of any unnecessary generalization. Rather, we will focus on a particular, yet meaningful, question which can be resumed as follows:

Given a concrete fractional Sobolev space of functions vanishing "at the boundary" of a set, does it coincide with an interpolation space?

We can already anticipate the conclusions of the paper and say that this is not always true. Let us now try to enter more in the details of the present paper.

Our concerns involve the so-called homogeneous fractional Sobolev-Slobodeckiu spaces $\mathcal{D}_{0}^{s, p}(\Omega)$. Given an open set $\Omega \subset \mathbb{R}^{N}$, an exponent $1 \leq p<\infty$ and a parameter $0<s<1$, this space is defined as the completion of $C_{0}^{\infty}(\Omega)$ with respect to the norm

$$
u \mapsto[u]_{W^{s, p}\left(\mathbb{R}^{N}\right)}:=\left(\iint_{\mathbb{R}^{N} \times \mathbb{R}^{N}} \frac{|u(x)-u(y)|^{p}}{|x-y|^{N+s p}} \mathrm{~d} x \mathrm{~d} y\right)^{\frac{1}{p}} .
$$

Such a space is the natural fractional counterpart of the homogeneous Sobolev space $\mathcal{D}_{0}^{1, p}(\Omega)$, defined as the completion of $C_{0}^{\infty}(\Omega)$ with respect to the norm

$$
u \mapsto\left(\int_{\Omega}|\nabla u|^{p} \mathrm{~d} x\right)^{\frac{1}{p}}
$$

The space $\mathcal{D}_{0}^{1, p}(\Omega)$ has been first studied by Deny and Lions in [13], among others. We recall that $\mathcal{D}_{0}^{1, p}(\Omega)$ is a natural setting for studying variational problems of the type

$$
\inf \left\{\frac{1}{p} \int_{\Omega}|\nabla u|^{p} \mathrm{~d} x-\int_{\Omega} f u \mathrm{~d} x\right\},
$$

supplemented with Dirichlet boundary conditions, in the absence of regularity assumptions on the boundary $\partial \Omega$. In the same way, the space $\mathcal{D}_{0}^{s, p}(\Omega)$ is the natural framework for studying minimization problems containing functionals of the type 


$$
\frac{1}{p} \iint_{\mathbb{R}^{N} \times \mathbb{R}^{N}} \frac{|u(x)-u(y)|^{p}}{|x-y|^{N+s p}} \mathrm{~d} x \mathrm{~d} y-\int_{\Omega} f u \mathrm{~d} x,
$$

in the presence of nonlocal Dirichlet boundary conditions, i.e., the values of $u$ are prescribed on the whole complement $\mathbb{R}^{N} \backslash \Omega$. Observe that even if this kind of boundary conditions may look weird, these are the correct ones when dealing with energies (1.1), which take into account interactions "from infinity."

The connection between the two spaces $\mathcal{D}_{0}^{1, p}(\Omega)$ and $\mathcal{D}_{0}^{s, p}(\Omega)$ is better appreciated by recalling that for $u \in C_{0}^{\infty}(\Omega)$, we have (see [5] and [26, Corollary 1.3])

$$
\lim _{s \nearrow 1}(1-s) \iint_{\mathbb{R}^{N} \times \mathbb{R}^{N}} \frac{|u(x)-u(y)|^{p}}{|x-y|^{N+s p}} \mathrm{~d} x \mathrm{~d} y=\alpha_{N, p} \int_{\Omega}|\nabla u|^{p} \mathrm{~d} x,
$$

with

$$
\alpha_{N, p}=\frac{1}{p} \int_{\mathbb{S}^{N-1}}\left|\left\langle\omega, \mathbf{e}_{1}\right\rangle\right|^{p} d \mathcal{H}^{N-1}(\omega), \quad \mathbf{e}_{1}=(1,0, \ldots, 0) .
$$

On the other hand, as $s \searrow 0$ we have (see [24, Theorem 3])

$$
\lim _{s \searrow 0} s \iint_{\mathbb{R}^{N} \times \mathbb{R}^{N}} \frac{|u(x)-u(y)|^{p}}{|x-y|^{N+s p}} \mathrm{~d} x \mathrm{~d} y=\beta_{N, p} \int_{\Omega}|u|^{p} \mathrm{~d} x,
$$

with

$$
\beta_{N, p}=\frac{2 N \omega_{N}}{p}
$$

and $\omega_{N}$ is the volume of the $N$-dimensional unit ball. These two results reflect the "interpolative" nature of the space $\mathcal{D}_{0}^{s, p}(\Omega)$, which will be, however, discussed in more detail in the sequel.

Indeed, one of our goals is to determine whether $\mathcal{D}_{0}^{s, p}(\Omega)$ coincides or not with the real interpolation space $\mathcal{X}_{0}^{s, p}(\Omega)$ defined as the completion of $C_{0}^{\infty}(\Omega)$ with respect to the norm

$$
\|u\|_{\mathcal{X}_{0}^{s, p}(\Omega)}:=\left(\int_{0}^{+\infty}\left(\frac{K\left(t, u, L^{p}(\Omega), \mathcal{D}_{0}^{1, p}(\Omega)\right)}{t^{s}}\right)^{p} \frac{\mathrm{d} t}{t}\right)^{\frac{1}{p}} .
$$

Here $K\left(t, \cdot L^{p}(\Omega), \mathcal{D}_{0}^{1, p}(\Omega)\right)$ is the $K$-functional associated with the spaces $L^{p}(\Omega)$ and $\mathcal{D}_{0}^{1, p}(\Omega)$, see Sect. 3 for more details.

In particular, we will be focused on obtaining double-sided norm inequalities leading to answer our initial question, i.e., estimates of the form

$$
\frac{1}{C}[u]_{W^{s, p}\left(\mathbb{R}^{N}\right)} \leq\|u\|_{\mathcal{X}_{0}^{s, p}(\Omega)} \leq C[u]_{W^{s, p}\left(\mathbb{R}^{N}\right)}, \quad u \in C_{0}^{\infty}(\Omega) .
$$

Moreover, we compute carefully the dependence on the parameter $s$ of the constant $C$. Indeed, we will see that $C$ can be taken independent of $s$.

\subsection{Results}

We now list the main achievements of our discussion:

1. the space $\mathcal{D}_{0}^{s, p}(\Omega)$ is always larger than $\mathcal{X}_{0}^{s, p}(\Omega)$ (see Proposition 4.1) and they do not coincide for general open sets, as we exhibit with an explicit example (see Example 4.4); 
2. they actually coincide on a large class of domains, i.e., bounded convex sets (Theorem 4.7), convex cones (Corollary 4.8), Lipschitz sets (Theorem 4.10);

3. the Poincare constants for the embeddings

$$
\mathcal{D}_{0}^{s, p}(\Omega) \hookrightarrow L^{p}(\Omega) \text { and } \mathcal{D}_{0}^{1, p}(\Omega) \hookrightarrow L^{p}(\Omega),
$$

are equivalent for the classes of sets at point 2 (Theorem 6.1). More precisely, by setting

$$
\lambda_{p}^{s}(\Omega)=\inf _{u \in C_{0}^{\infty}(\Omega)}\left\{[u]_{W^{s, p}\left(\mathbb{R}^{N}\right)}^{p}:\|u\|_{L^{p}(\Omega)}=1\right\}, \quad 0<s<1,
$$

and

$$
\lambda_{p}^{1}(\Omega)=\inf _{u \in C_{0}^{\infty}(\Omega)}\left\{\int_{\Omega}|\nabla u|^{p} \mathrm{~d} x:\|u\|_{L^{p}(\Omega)}=1\right\}
$$

we have

$$
\frac{1}{C}\left(\lambda_{p}^{1}(\Omega)\right)^{s} \leq s(1-s) \lambda_{p}^{s}(\Omega) \leq C\left(\lambda_{p}^{1}(\Omega)\right)^{s}
$$

Moreover, on convex sets the constant $C>0$ entering in the relevant estimate is universal, i.e., it depends on $N$ and $p$ only. On the other hand, we show that this equivalence fails if we drop any kind of regularity assumptions on the sets (see Remark 6.3).

As a byproduct of our discussion, we also highlight some weird and unnatural behaviors of the interpolation space $\mathcal{X}_{0}^{s, p}(\Omega)$ :

- the "extension by zero" operator $\mathcal{X}_{0}^{s, p}(\Omega) \hookrightarrow \mathcal{X}_{0}^{s, p}\left(\mathbb{R}^{N}\right)$ is not an isometry for general open sets (see Remark 4.6) and the two norms

$$
\|\cdot\|_{\mathcal{X}_{0}^{s, p}(\Omega)} \text { and }\|\cdot\|_{\mathcal{X}_{0}^{s, p}\left(\mathbb{R}^{N}\right)},
$$

may not be equivalent on $C_{0}^{\infty}(\Omega)$. This is in contrast with what happens for the spaces $L^{p}(\Omega), \mathcal{D}_{0}^{1, p}(\Omega)$ and $\mathcal{D}_{0}^{s, p}(\Omega)$;

- the sharp Poincaré interpolation constant

$$
\Lambda_{p}^{s}(\Omega)=\inf _{u \in C_{0}^{\infty}(\Omega)}\left\{\|u\|_{\mathcal{X}_{0}^{s, p}(\Omega)}^{p}:\|u\|_{L^{p}(\Omega)}=1\right\}, \quad 0<s<1
$$

is sensitive to removing sets with zero capacity. In other words, if we remove a compact set $E \Subset \Omega$ having zero capacity in the sense of $\mathcal{X}_{0}^{s, p}(\Omega)$, it may happen that (see Lemma 5.4)

$$
\Lambda_{p}^{s}(\Omega \backslash E)>\Lambda_{p}^{s}(\Omega) .
$$

Again, this is in contrast with the case of $\mathcal{D}_{0}^{1, p}(\Omega)$ and $\mathcal{D}_{0}^{s, p}(\Omega)$.

Remark 1.1 As recalled at the beginning, nowadays there is a huge literature on Sobolev spaces of fractional order. Nevertheless, to the best of our knowledge, a detailed discussion on the space $\mathcal{D}_{0}^{s, p}(\Omega)$ in connection with interpolation theory seems to be missing. For this reason, we believe that our discussion is of independent interest.

We also point out that for Sobolev spaces of functions not necessarily vanishing at the boundary, there is a very nice paper [11] by Chandler-Wilde, Hewett and Moiola comparing "concrete" constructions with the interpolation one. 


\subsection{Plan of the paper}

In Sect. 2 we present the relevant Sobolev spaces, constructed with the concrete approach based on the so-called Sobolev-Slobodeckil norms. Then in Sect. 3 we introduce the homogeneous interpolation space we want to work with. Essentially, no previous knowledge of interpolation theory is necessary.

The comparison between the concrete space and the interpolation one is contained in Sect. 4. This in turn is divided in three subsections, each one dealing with a different class of open sets. We point out here that we preferred to treat convex sets separately from Lipschitz sets, for two reasons: the first one is that for convex sets the comparison between the two spaces can be done "by hands," without using any extension theorem. This in turn permits to have a better control on the relevant constants entering in the estimates. The second one is that in proving the result for Lipschitz sets, we actually use the result for convex sets.

In order to complement the comparison between the two spaces, in Sect. 5 we compare the two relevant notions of capacity, naturally associated with the norms of these spaces. Finally, Sect. 6 compares the Poincaré constants.

The paper ends with three appendices: the first one contains the construction of a counterexample used throughout the whole paper; the second one proves a version of the one-dimensional Hardy inequality; and the last one contains a geometric expedient result for convex sets.

\section{Preliminaries}

\subsection{Basic notation}

In what follows, we will always denote by $N$ the dimension of the ambient space. For an open set $\Omega \subset \mathbb{R}^{N}$, we indicate by $|\Omega|$ its $N$-dimensional Lebesgue measure. The symbol $\mathcal{H}^{k}$ will stand for the $k$-dimensional Hausdorff measure. Finally, we set

$$
B_{R}\left(x_{0}\right)=\left\{x \in \mathbb{R}^{N}:\left|x-x_{0}\right|<R\right\},
$$

and

$$
\omega_{N}=\left|B_{1}(0)\right|
$$

\subsection{Sobolev spaces}

For $1 \leq p<\infty$ and an open set $\Omega \subset \mathbb{R}^{N}$, we use the classical definition

$$
W^{1, p}(\Omega):=\left\{u \in L^{p}(\Omega): \int_{\Omega}|\nabla u|^{p} \mathrm{~d} x<+\infty\right\} .
$$

This is a Banach space endowed with the norm

$$
\|u\|_{W^{1, p}(\Omega)}=\left(\|u\|_{L^{p}(\Omega)}^{p}+\|\nabla u\|_{L^{p}(\Omega)}^{p}\right)^{\frac{1}{p}} .
$$

We also denote by $\mathcal{D}_{0}^{1, p}(\Omega)$ the homogeneous Sobolev space, defined as the completion of $C_{0}^{\infty}(\Omega)$ with respect to the norm

$$
u \mapsto\|\nabla u\|_{L^{p}(\Omega)} .
$$


If the open set $\Omega \subset \mathbb{R}^{N}$ supports the classical Poincaré inequality

$$
c \int_{\Omega}|u|^{p} \mathrm{~d} x \leq \int_{\Omega}|\nabla u|^{p} \mathrm{~d} x, \quad \text { for every } u \in C_{0}^{\infty}(\Omega),
$$

then $\mathcal{D}_{0}^{1, p}(\Omega)$ is indeed a functional space and it coincides with the closure in $W^{1, p}(\Omega)$ of $C_{0}^{\infty}(\Omega)$. We will set

$$
\lambda_{p}^{1}(\Omega)=\inf _{u \in C_{0}^{\infty}(\Omega)}\left\{\|\nabla u\|_{L^{p}(\Omega)}^{p}:\|u\|_{L^{p}(\Omega)}=1\right\} .
$$

It occurs $\lambda_{p}^{1}(\Omega)=0$ whenever $\Omega$ does not support such a Poincaré inequality.

Remark 2.1 We remark that one could also consider the space

$$
W_{0}^{1, p}(\Omega):=\left\{u \in W^{1, p}\left(\mathbb{R}^{N}\right): u=0 \text { a.e. in } \mathbb{R}^{N} \backslash \Omega\right\} .
$$

It is easy to see that $\mathcal{D}_{0}^{1, p}(\Omega) \subset W_{0}^{1, p}(\Omega)$, whenever $\mathcal{D}_{0}^{1, p}(\Omega) \hookrightarrow L^{p}(\Omega)$. If in addition $\partial \Omega$ is continuous, then both spaces are known to coincide, thanks to the density of $C_{0}^{\infty}(\Omega)$ in $W_{0}^{1, p}(\Omega)$, see [20, Theorem 1.4.2.2].

\subsection{A homogeneous Sobolev-Slobodecki space}

Given $0<s<1$ and $1 \leq p<\infty$, the fractional Sobolev space $W^{s, p}\left(\mathbb{R}^{N}\right)$ is defined as

$$
W^{s, p}\left(\mathbb{R}^{N}\right):=\left\{u \in L^{p}\left(\mathbb{R}^{N}\right):[u]_{W^{s, p}\left(\mathbb{R}^{N}\right)}<+\infty\right\},
$$

where the Sobolev-Slobodeckil seminorm $[\cdot]_{W^{s, p}\left(\mathbb{R}^{N}\right)}$ is defined as

$$
[u]_{W^{s, p}\left(\mathbb{R}^{N}\right)}:=\left(\iint_{\mathbb{R}^{N} \times \mathbb{R}^{N}} \frac{|u(x)-u(y)|^{p}}{|x-y|^{N+s p}} \mathrm{~d} x \mathrm{~d} y\right)^{\frac{1}{p}} .
$$

This is a Banach space endowed with the norm

$$
\|u\|_{W^{s, p}\left(\mathbb{R}^{N}\right)}=\left(\|u\|_{L^{p}\left(\mathbb{R}^{N}\right)}^{p}+[u]_{W^{s, p}\left(\mathbb{R}^{N}\right)}^{p}\right)^{\frac{1}{p}} .
$$

In what follows, we need to consider nonlocal homogeneous Dirichlet boundary conditions, outside an open set $\Omega \subset \mathbb{R}^{N}$. In this setting, it is customary to consider the homogeneous Sobolev-Slobodeckiu space $\mathcal{D}_{0}^{s, p}(\Omega)$. The latter is defined as the completion of $C_{0}^{\infty}(\Omega)$ with respect to the norm

$$
u \mapsto[u]_{W^{s, p}\left(\mathbb{R}^{N}\right)} .
$$

Observe that the latter is indeed a norm on $C_{0}^{\infty}(\Omega)$. Whenever the open set $\Omega \subset \mathbb{R}^{N}$ admits the following Poincaré inequality

$$
c \int_{\Omega}|u|^{p} \mathrm{~d} x \leq \iint_{\mathbb{R}^{N} \times \mathbb{R}^{N}} \frac{|u(x)-u(y)|^{p}}{|x-y|^{N+s p}} \mathrm{~d} x \mathrm{~d} y, \quad \text { for every } u \in C_{0}^{\infty}(\Omega),
$$

we get that $\mathcal{D}_{0}^{s, p}(\Omega)$ is a functional space continuously embedded in $L^{p}(\Omega)$. In this case, it coincides with the closure in $W^{s, p}\left(\mathbb{R}^{N}\right)$ of $C_{0}^{\infty}(\Omega)$. We endow the space $\mathcal{D}_{0}^{s, p}(\Omega)$ with the norm

$$
\|u\|_{\mathcal{D}_{0}^{s, p}(\Omega)}:=[u]_{W^{s, p}\left(\mathbb{R}^{N}\right)} .
$$


We also define

$$
\lambda_{p}^{s}(\Omega)=\inf _{u \in C_{0}^{\infty}(\Omega)}\left\{\|u\|_{\mathcal{D}_{0}^{s, p}(\Omega)}^{p}:\|u\|_{L^{p}(\Omega)}=1\right\},
$$

i.e., this is the sharp constant in the relevant Poincaré inequality. Some embedding properties of the space $\mathcal{D}_{0}^{s, p}(\Omega)$ are investigated in [18].

Remark 2.2 As in the local case, one could also consider the space

$$
W_{0}^{s, p}(\Omega):=\left\{u \in W^{s, p}\left(\mathbb{R}^{N}\right): u=0 \text { a.e. in } \mathbb{R}^{N} \backslash \Omega\right\} .
$$

It is easy to see that $\mathcal{D}_{0}^{s, p}(\Omega) \subset W_{0}^{s, p}(\Omega)$, whenever $\mathcal{D}_{0}^{s, p}(\Omega) \hookrightarrow L^{p}(\Omega)$. As before, if $\partial \Omega$ is continuous, then both spaces are known to coincide, again thanks to the density of $C_{0}^{\infty}(\Omega)$ in $W_{0}^{s, p}(\Omega)$, see [20, Theorem 1.4.2.2].

\subsection{Another space of functions vanishing at the boundary}

Another natural fractional Sobolev space of functions "vanishing at the boundary" is given by the completion of $C_{0}^{\infty}(\Omega)$ with respect to the localized norm

$$
[u]_{W^{s, p}(\Omega)}=\left(\iint_{\Omega \times \Omega} \frac{|u(x)-u(y)|^{p}}{|x-y|^{N+s p}} \mathrm{~d} x \mathrm{~d} y\right)^{\frac{1}{p}} .
$$

We will denote this space by $\stackrel{\circ}{D}^{s, p}(\Omega)$ and endow it with the norm $\left[\|u\|_{D^{s, p}(\Omega)}:=[u]_{W^{s, p}(\Omega)}\right]$. We recall the following

Lemma 2.3 Let $1<p<\infty$ and $0<s<1$. For every $\Omega \subset \mathbb{R}^{N}$ open bounded Lipschitz set, we have:

- if $s p>1$, then

$$
\mathcal{D}_{0}^{s, p}(\Omega)=\stackrel{\circ}{D}^{s, p}(\Omega) ;
$$

- if $s \quad$, then there exists a sequence $\left\{u_{n}\right\}_{n \in \mathbb{N}} \subset C_{0}^{\infty}(\Omega)$ such that

$$
\lim _{n \rightarrow \infty} \frac{\left\|u_{n}\right\|_{D^{s, p}(\Omega)}}{\left\|u_{n}\right\|_{\mathcal{D}_{0}^{s, p}(\Omega)}}=0 .
$$

Proof The proof of the first fact is contained in [7, Proposition B.1].

As for the case $s p \leq 1$, in [15, Section 2] Dyda constructed a sequence $\left\{u_{n}\right\}_{n \in \mathbb{N}} \subset C_{0}^{\infty}(\Omega)$ such that

$$
\lim _{n \rightarrow \infty}\left\|u_{n}\right\|_{D^{s, p}(\Omega)}=0 \text { and } \lim _{n \rightarrow \infty}\left\|u_{n}-1_{\Omega}\right\|_{L^{p}(\Omega)}=0 .
$$

By observing that for such a sequence we have

$$
\lim _{n \rightarrow \infty}\left\|u_{n}\right\|_{\mathcal{D}_{0}^{s, p}(\Omega)} \geq\left(\lambda_{p}^{s}(\Omega)\right)^{\frac{1}{p}} \lim _{n \rightarrow \infty}\left\|u_{n}\right\|_{L^{p}(\Omega)}=\left(\lambda_{p}^{s}(\Omega)|\Omega|\right)^{\frac{1}{p}},
$$

we get the desired conclusion, by observing that $\lambda_{p}^{s}(\Omega)>0$ for an open bounded set, thanks to [8, Corollary 5.2]. 
Remark 2.4 Clearly, we always have

$$
\|u\|_{D^{s, p}(\Omega)} \leq\|u\|_{\mathcal{D}_{0}^{s, p}(\Omega)}, \quad \text { for every } u \in C_{0}^{\infty}(\Omega) .
$$

As observed in [16], the reverse inequality

$$
\|u\|_{\mathcal{D}_{0}^{s, p}(\Omega)} \leq C\|u\|_{D^{s, p}(\Omega)}, \quad \text { for every } u \in C_{0}^{\infty}(\Omega),
$$

is equivalent to the validity of the Hardy-type inequality

$$
\int_{\Omega}|u(x)|^{p}\left(\int_{\mathbb{R}^{N} \backslash \Omega}|x-y|^{-N-s p} y\right) \mathrm{d} x \leq C \iint_{\Omega \times \Omega} \frac{|u(x)-u(y)|^{p}}{|x-y|^{N+s p}} \mathrm{~d} x \mathrm{~d} y .
$$

A necessary and sufficient condition for this to happen is proved in [16, Proposition 2]. We also observe that the failure of (2.1) implies that in general the "extension by zero" operator

$$
\mathcal{T}_{0}: \stackrel{\circ}{D}^{s, p}(\Omega) \rightarrow \stackrel{\circ}{D}^{s, p}\left(\mathbb{R}^{N}\right),
$$

is not continuous. We refer to [16] for a detailed discussion of this issue.

Remark 2.5 The space $\stackrel{\circ}{D}^{s, p}(\Omega)$ is quite problematic in general, especially in the case $s p \leq 1$ where it may fail to be a functional space. A more robust variant of this space is

$$
\widetilde{D}^{s, p}(\Omega)=\text { "closure of } C_{0}^{\infty}(\Omega) \text { in } W^{s, p}(\Omega) \text { ". }
$$

By definition, this is automatically a functional space, continuously contained in $W^{s, p}(\Omega)$. It is a classical fact that if $\Omega$ is a bounded open set with smooth boundary, then

$$
\widetilde{D}^{s, p}(\Omega)=W^{s, p}(\Omega), \text { for } s p<1,
$$

see [32, Theorem 3.4.3]. Moreover, we also have

$$
\widetilde{D}^{s, p}(\Omega)=\mathcal{D}_{0}^{s, p}(\Omega), \text { for } s p \neq 1,
$$

see, for example, [7, Proposition B.1].

\section{An interpolation space}

Let $\Omega \subset \mathbb{R}^{N}$ be an open set. If $X(\Omega)$ and $Y(\Omega)$ are two normed vector spaces containing $C_{0}^{\infty}(\Omega)$ as a dense subspace, we define for every $t>0$ and $u \in C_{0}^{\infty}(\Omega)$ the $K$-functional

$$
K(t, u, X(\Omega), Y(\Omega)):=\inf _{v \in C_{0}^{\infty}(\Omega)}\left\{\|u-v\|_{X(\Omega)}+t\|v\|_{Y(\Omega)}\right\} .
$$

We are interested in the following specific case: let us take $0<s<1$ and $1<p<\infty$, we choose

$$
X(\Omega)=L^{p}(\Omega) \text { and } Y(\Omega)=\mathcal{D}_{0}^{1, p}(\Omega) .
$$

Then we use the notation

$$
\|u\|_{\mathcal{X}_{0}^{s, p}(\Omega)}:=\left(\int_{0}^{+\infty}\left(\frac{K\left(t, u, L^{p}(\Omega), \mathcal{D}_{0}^{1, p}(\Omega)\right)}{t^{s}}\right)^{p} \frac{\mathrm{d} t}{t}\right)^{\frac{1}{p}}, \quad u \in C_{0}^{\infty}(\Omega) .
$$

It is standard to see that this is a norm on $C_{0}^{\infty}(\Omega)$, see [4, Section 3.1]. We will indicate by $\mathcal{X}_{0}^{s, p}(\Omega)$ the completion of $C_{0}^{\infty}(\Omega)$ with respect to this norm. 
The first result is the Poincaré inequality for the interpolation space $\mathcal{X}_{0}^{s, p}(\Omega)$. The main focus is on the explicit dependence of the constant on the local Poincaré constant $\lambda_{p}^{1}$.

Lemma 3.1 Let $1<p<\infty$ and $0<s<1$. Let $\Omega \subset \mathbb{R}^{N}$ be an open set. Then for every $u \in C_{0}^{\infty}(\Omega)$ we have

$$
\left(\lambda_{p}^{1}(\Omega)\right)^{s}\|u\|_{L^{p}(\Omega)}^{p} \leq p s(1-s)\|u\|_{\mathcal{X}_{0}^{s, p}(\Omega)}^{p} .
$$

Proof We proceed in two stages: we first prove that

$$
\|u\|_{L^{p}(\Omega)}^{p} \lesssim \int_{0}^{+\infty}\left(\frac{K\left(t, u, L^{p}(\Omega), L^{p}(\Omega)\right)}{t^{s}}\right)^{p} \frac{\mathrm{d} t}{t},
$$

and then we show that the last integral is estimated from the above by the norm $\mathcal{X}_{0}^{s, p}(\Omega)$.

First stage Let us take $u \in C_{0}^{\infty}(\Omega)$, for every $t \geq 1$ and $v \in C_{0}^{\infty}(\Omega)$

$$
\|u\|_{L^{p}(\Omega)} \leq\|u-v\|_{L^{p}(\Omega)}+t\|v\|_{L^{p}(\Omega)} .
$$

By taking the infimum, we thus get

$$
\|u\|_{L^{p}(\Omega)} \leq K\left(t, u, L^{p}(\Omega), L^{p}(\Omega)\right) .
$$

By integrating with respect to the singular measure $\mathrm{d} t / t$, we then get

$$
\int_{1}^{+\infty}\left(\frac{K\left(t, u, L^{p}(\Omega), L^{p}(\Omega)\right)}{t^{s}}\right)^{p} \frac{\mathrm{d} t}{t} \geq \int_{1}^{+\infty} t^{-s p}\|u\|_{L^{p}(\Omega)}^{p} \frac{\mathrm{d} t}{t}=\frac{\|u\|_{L^{p}(\Omega)}^{p}}{s p} .
$$

We now pick $0<t<1$, by triangle inequality we get for every $v \in C_{0}^{\infty}(\Omega)$

$$
\begin{aligned}
t\|u\|_{L^{p}(\Omega)} & \leq t\|u-v\|_{L^{p}(\Omega)}+t\|v\|_{L^{p}(\Omega)} \\
& \leq\|u-v\|_{L^{p}(\Omega)}+t\|v\|_{L^{p}(\Omega)} .
\end{aligned}
$$

By taking the infimum over $v \in C_{0}^{\infty}(\Omega)$, we obtain for $u \in C_{0}^{\infty}(\Omega)$ and $0<t<1$

$$
t\|u\|_{L^{p}(\Omega)} \leq K\left(t, u, L^{p}(\Omega), L^{p}(\Omega)\right) .
$$

By integrating again, we get this time

$$
\int_{0}^{1}\left(\frac{K\left(t, u, L^{p}(\Omega), L^{p}(\Omega)\right)}{t^{s}}\right)^{p} \frac{\mathrm{d} t}{t} \geq \int_{0}^{1} t^{p-s p}\|u\|_{L^{p}(\Omega)}^{p} \frac{\mathrm{d} t}{t}=\frac{\|u\|_{L^{p}(\Omega)}^{p}}{(1-s) p} .
$$

By summing up (3.3) and (3.4), we get the estimate

$$
\|u\|_{L^{p}(\Omega)}^{p} \leq p s(1-s) \int_{0}^{+\infty}\left(\frac{K\left(t, u, L^{p}(\Omega), L^{p}(\Omega)\right)}{t^{s}}\right)^{p} \frac{\mathrm{d} t}{t} .
$$

Second stage Given $u \in C_{0}^{\infty}(\Omega)$, we take $v \in C_{0}^{\infty}(\Omega)$. We can suppose that $\lambda_{p}^{1}(\Omega)>0$; otherwise, (3.2) trivially holds. By definition of $\lambda_{p}^{1}(\Omega)$, we have that

$$
\|u-v\|_{L^{p}(\Omega)}+t\|v\|_{L^{p}(\Omega)} \leq\|u-v\|_{L^{p}(\Omega)}+t\left(\lambda_{p}^{1}(\Omega)\right)^{-\frac{1}{p}}\|\nabla v\|_{L^{p}(\Omega)} .
$$

If we recall the definition (3.1) of the $K$-functional, we get

$$
K\left(t, u, L^{p}(\Omega), L^{p}(\Omega)\right)^{p} \leq\left(\|u-v\|_{L^{p}(\Omega)}+\frac{t}{\left(\lambda_{p}^{1}(\Omega)\right)^{\frac{1}{p}}}\|\nabla v\|_{L^{p}(\Omega)}\right)^{p},
$$


and by taking infimum over $v \in C_{0}^{\infty}(\Omega)$ and multiplying by $t^{-s p}$, we get

$$
t^{-s p} K\left(t, u, L^{p}(\Omega), L^{p}(\Omega)\right)^{p} \leq t^{-s p} K\left(\frac{t}{\left(\lambda_{p}^{1}(\Omega)\right)^{\frac{1}{p}}}, u, L^{p}(\Omega), \mathcal{D}_{0}^{1, p}(\Omega)\right)^{p} .
$$

We integrate over $t>0$, by performing the change of variable $\tau=t /\left(\lambda_{p}^{1}(\Omega)\right)^{\frac{1}{p}}$ we get

$$
\int_{0}^{+\infty}\left(\frac{K\left(t, u, L^{p}(\Omega), L^{p}(\Omega)\right)}{t^{s}}\right)^{p} \frac{\mathrm{d} t}{t} \leq \frac{1}{\left(\lambda_{p}^{1}(\Omega)\right)^{s}}\|u\|_{\mathcal{X}_{0}^{s, p}(\Omega)}^{p} .
$$

By using this in (3.5), we prove the desired inequality (3.2).

We will set

$$
\Lambda_{p}^{s}(\Omega)=\inf _{u \in C_{0}^{\infty}(\Omega)}\left\{\|u\|_{\mathcal{X}_{0}^{s, p}(\Omega)}^{p}:\|u\|_{L^{p}(\Omega)}=1\right\},
$$

i.e., this is the sharp constant in the relevant Poincaré inequality. As a consequence of (3.2), we obtain

$$
\left(\lambda_{p}^{1}(\Omega)\right)^{s} \leq p s(1-s) \Lambda_{p}^{s}(\Omega)
$$

Proposition 3.2 (Interpolation inequality) Let $1<p<\infty$ and $0<s<1$. Let $\Omega \subset \mathbb{R}^{N}$ be an open set. For every $u \in C_{0}^{\infty}(\Omega)$ we have

$$
p s(1-s)\|u\|_{\mathcal{X}_{0}^{s, p}(\Omega)}^{p} \leq\|u\|_{L^{p}(\Omega)}^{p(1-s)}\|\nabla u\|_{L^{p}(\Omega)}^{s p} .
$$

In particular, we also obtain

$$
p s(1-s) \Lambda_{p}^{s}(\Omega) \leq\left(\lambda_{p}^{1}(\Omega)\right)^{s} .
$$

Proof We can assume that $u \not \equiv 0$; otherwise, there is nothing prove. In the definition of the $K$-functional $K\left(t, u, L^{p}(\Omega), \mathcal{D}_{0}^{1, p}(\Omega)\right)$, we take $v=\tau u$ for $\tau>0$; thus, we obtain

$$
\begin{aligned}
K\left(t, u, L^{p}(\Omega), \mathcal{D}_{0}^{1, p}(\Omega)\right) & \leq \inf _{\tau>0}\left[|1-\tau|\|u\|_{L^{p}(\Omega)}+t \tau\|\nabla u\|_{L^{p}(\Omega)}\right] \\
& =\min \left\{\|u\|_{L^{p}(\Omega)}, t\|\nabla u\|_{L^{p}(\Omega)}\right\} .
\end{aligned}
$$

By raising to the power $p$ and integrating for $t>0$, we get

$$
\begin{aligned}
& \|u\|_{\mathcal{X}_{0}^{s, p}(\Omega)}^{p} \leq \int_{0}^{+\infty} \frac{\min \left\{\|u\|_{L^{p}(\Omega)}^{p}, t^{p}\|\nabla u\|_{L^{p}(\Omega)}^{p}\right\}}{t^{s p}} \frac{\mathrm{d} t}{t}
\end{aligned}
$$

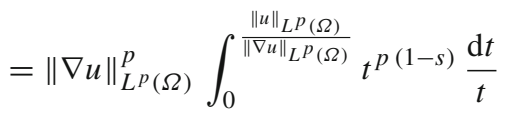

$$
\begin{aligned}
& +\|u\|_{L^{p}(\Omega)}^{p} \int_{\frac{\|u\|_{L^{p}(\Omega)}}{\|\nabla u\|_{L^{p}(\Omega)}}}^{+\infty} t^{-s p} \frac{\mathrm{d} t}{t} \\
& =\|u\|_{L^{p}(\Omega)}^{p(1-s)}\|\nabla u\|_{L^{p}(\Omega)}^{s p}\left[\frac{1}{p(1-s)}+\frac{1}{s p}\right] .
\end{aligned}
$$

We thus get the desired conclusion (3.7). The estimate (3.8) easily follows from the definition of Poincaré constant. 
From (3.6) and (3.8), we get, in particular, the following

Corollary 3.3 (Equivalence of Poincaré constants) Let $1<p<\infty$ and $0<s<1$. For every $\Omega \subset \mathbb{R}^{N}$ open set we have

$$
p s(1-s) \Lambda_{p}^{s}(\Omega)=\left(\lambda_{p}^{1}(\Omega)\right)^{s} .
$$

In particular, there holds

$$
\mathcal{D}_{0}^{1, p}(\Omega) \hookrightarrow L^{p}(\Omega) \Longleftrightarrow \mathcal{X}_{0}^{s, p}(\Omega) \hookrightarrow L^{p}(\Omega) .
$$

Remark 3.4 (Extensions by zero in $\mathcal{X}_{0}^{s, p}$ ) We observe that by interpolating the "extension by zero" operators

$$
\mathcal{T}_{0}: \mathcal{D}_{0}^{1, p}(\Omega) \rightarrow \mathcal{D}_{0}^{1, p}\left(\mathbb{R}^{N}\right) \text { and } \mathcal{T}_{0}: L^{p}(\Omega) \rightarrow L^{p}\left(\mathbb{R}^{N}\right),
$$

which are both continuous, one obtains the same result for the interpolating spaces. In other words, we have

$$
\|u\|_{\mathcal{X}_{0}^{s, p}\left(\mathbb{R}^{N}\right)}^{p} \leq\|u\|_{\mathcal{X}_{0}^{s, p}(\Omega)}^{p}, \quad \text { for every } u \in C_{0}^{\infty}(\Omega) .
$$

This can be also seen directly: it is sufficient to observe that $C_{0}^{\infty}(\Omega) \subset C_{0}^{\infty}\left(\mathbb{R}^{N}\right)$; thus, we immediately get

$$
K\left(t, u, L^{p}\left(\mathbb{R}^{N}\right), \mathcal{D}_{0}^{1, p}\left(\mathbb{R}^{N}\right)\right) \leq K\left(t, u, L^{p}(\Omega), \mathcal{D}_{0}^{1, p}(\Omega)\right),
$$

since in the $K$-functional on the left-hand side the infimum is performed on a larger class. By integrating, we get the conclusion.

However, differently from the case of $\mathcal{D}_{0}^{1, p}(\Omega), L^{p}(\Omega)$ and $\mathcal{D}_{0}^{s, p}(\Omega)$, in general for $u \in C_{0}^{\infty}(\Omega)$ we have

$$
\|u\|_{\mathcal{X}_{0}^{s, p}\left(\mathbb{R}^{N}\right)}^{p}<\|u\|_{\mathcal{X}_{0}^{s, p}(\Omega)}^{p} .
$$

In other words, even if $u \equiv 0$ outside $\Omega$, passing from $\Omega$ to $\mathbb{R}^{N}$ has an impact on the interpolation norm.

Actually, if $\Omega$ has not smooth boundary, the situation can be much worse than this. We refer to Remark 4.6.

\section{Interpolation versus Sobolev-Slobodeckii}

\subsection{General sets}

We want to compare the norms of $\mathcal{D}_{0}^{s, p}(\Omega)$ and $\mathcal{X}_{0}^{s, p}(\Omega)$. We start with the simplest estimate, which is valid for every open set.

Proposition 4.1 (Comparison of norms I) Let $1<p<\infty$ and $0<s<1$. Let $\Omega \subset \mathbb{R}^{N}$ be an open set, then for every $u \in C_{0}^{\infty}(\Omega)$ we have

$$
\frac{1}{2^{p(1-s)} N \omega_{N}}\|u\|_{\mathcal{D}_{0}^{s, p}(\Omega)}^{p} \leq\|u\|_{\mathcal{X}_{0}^{s, p}(\Omega)}^{p} .
$$

In particular, we have the continuous inclusion $\mathcal{X}_{0}^{s, p}(\Omega) \subset \mathcal{D}_{0}^{s, p}(\Omega)$. 
Proof To prove (4.1), we take $h \in \mathbb{R}^{N} \backslash\{0\}$ and $\varepsilon>0$, then there exists $v_{h, \varepsilon} \in C_{0}^{\infty}(\Omega)$ such that

$$
\left\|u-v_{h, \varepsilon}\right\|_{L^{p}(\Omega)}+\frac{|h|}{2}\left\|\nabla v_{h, \varepsilon}\right\|_{L^{p}(\Omega)} \leq(1+\varepsilon) K\left(\frac{|h|}{2}, u, L^{p}(\Omega), \mathcal{D}_{0}^{1, p}(\Omega)\right) .
$$

Thus, for $h \neq 0$ we get $^{1}$

$$
\begin{aligned}
\left(\int_{\mathbb{R}^{N}} \frac{|u(x+h)-u(x)|^{p}}{\left.|h|^{N+s p} \mathrm{~d} x\right)^{\frac{1}{p}} \leq}\right. & \left(\int_{\mathbb{R}^{N}} \frac{\left|u(x+h)-v_{h, \varepsilon}(x+h)-u(x)+v_{h, \varepsilon}(x)\right|^{p}}{|h|^{N+s} p} \mathrm{~d} x\right)^{\frac{1}{p}} \\
& +\left(\int_{\mathbb{R}^{N}} \frac{\left|v_{h, \varepsilon}(x+h)-v_{h, \varepsilon}(x)\right|^{p}}{|h|^{N+s} p} \mathrm{~d} x\right)^{\frac{1}{p}} \\
\leq & 2|h|^{-\frac{N}{p}-s}\left\|u-v_{h, \varepsilon}\right\|_{L^{p}(\Omega)} \\
& +|h|^{1-\frac{N}{p}-s}\left\|\nabla v_{h, \varepsilon}\right\|_{L^{p}(\Omega)} \\
\leq & 2|h|^{-\frac{N}{p}-s}\left(\left\|u-v_{h, \varepsilon}\right\|_{L^{p}(\Omega)}+\frac{|h|}{2}\left\|\nabla v_{h, \varepsilon}\right\|_{L^{p}(\Omega)}\right)
\end{aligned}
$$

By using (4.2), we then obtain

$$
\int_{\mathbb{R}^{N}} \frac{|u(x+h)-u(x)|^{p}}{|h|^{N+s} p} \mathrm{~d} x \leq 2^{p}(1+\varepsilon)^{p}\left(\frac{K\left(|h| / 2, u, L^{p}(\Omega), \mathcal{D}_{0}^{1, p}(\Omega)\right)}{|h|^{s}}\right)^{p} \frac{1}{|h|^{N}} .
$$

We now integrate with respect to $h \in \mathbb{R}^{N}$ and use spherical coordinates. This yields

$$
\begin{aligned}
& \iint_{\mathbb{R}^{N} \times \mathbb{R}^{N}} \frac{|u(x+h)-u(x)|^{p}}{|h|^{N+s p} \mathrm{~d} x \mathrm{~d} h} \\
& \quad \leq 2^{p}(1+\varepsilon)^{p} \int_{\mathbb{R}^{N}}\left(\frac{K\left(|h| / 2, u, L^{p}(\Omega), \mathcal{D}_{0}^{1, p}(\Omega)\right)}{|h|^{s}}\right)^{p} \frac{\mathrm{d} h}{|h|^{N}} \\
& \quad=2^{p}(1+\varepsilon)^{p} N \omega_{N} \int_{0}^{+\infty}\left(\frac{K\left(t / 2, u, L^{p}(\Omega), \mathcal{D}_{0}^{1, p}(\Omega)\right)}{t^{s}}\right)^{p} \frac{\mathrm{d} t}{t} .
\end{aligned}
$$

By making the change of variable $t / 2=\tau$ and exploiting the arbitrariness of $\varepsilon>0$, we eventually reach the desired estimate.

Corollary 4.2 (Interpolation inequality for $\mathcal{D}_{0}^{s, p}$ ) Let $1<p<\infty$ and $0<s<1$. Let $\Omega \subset \mathbb{R}^{N}$ be an open set. For every $u \in C_{0}^{\infty}(\Omega)$ we have

$$
s(1-s)\|u\|_{\mathcal{D}_{0}^{s, p}(\Omega)}^{p} \leq \frac{2^{p(1-s)} N \omega_{N}}{p}\|u\|_{L^{p}(\Omega)}^{p(1-s)}\|\nabla u\|_{L^{p}(\Omega)}^{s p} .
$$

1 In the second inequality, we use the classical fact

$$
\begin{aligned}
\int_{\mathbb{R}^{N}}|\varphi(x+h)-\varphi(x)|^{p} \mathrm{~d} x & =\int_{\mathbb{R}^{N}}\left|\int_{0}^{1}\langle\nabla \varphi(x+t h), h\rangle \mathrm{d} t\right|^{p} \mathrm{~d} x \\
& \leq|h|^{p} \int_{\mathbb{R}^{N}} \int_{0}^{1}|\nabla \varphi(x+t h)|^{p} \mathrm{~d} t \mathrm{~d} x \\
& =|h|^{p} \int_{0}^{1}\left(\int_{\mathbb{R}^{N}}|\nabla \varphi(x+t h)|^{p} \mathrm{~d} x\right) \mathrm{d} t=|h|^{p}\|\nabla \varphi\|_{L^{p}\left(\mathbb{R}^{N}\right)}
\end{aligned}
$$


Proof It is sufficient to combine Propositions 4.1 and 3.2.

Remark 4.3 For $p \searrow 1$, the previous inequality becomes [7, Proposition 4.2]. In this case, the constant in (4.3) is sharp for $N=1$.

For a general open set $\Omega \subset \mathbb{R}^{N}$, the converse of inequality (4.1) does not hold. This means that in general we have

$$
\mathcal{X}_{0}^{s, p}(\Omega) \subset \mathcal{D}_{0}^{s, p}(\Omega) \text { and } \mathcal{X}_{0}^{s, p}(\Omega) \neq \mathcal{D}_{0}^{s, p}(\Omega),
$$

the inclusion being continuous. We use the construction of "Appendix A", in order to give a counterexample.

Example 4.4 With the notation of "Appendix A", let us take ${ }^{2}$

$$
E=\mathbb{R}^{N} \backslash\left(\bigcup_{z \in \mathbb{Z}^{N}}(F+z)\right) \text {, with } F=\left[-\frac{1}{4}, \frac{1}{4}\right]^{N-1} \times\{0\} .
$$

For every $\varepsilon>0$, we take $u_{n} \in C_{0}^{\infty}\left(\widetilde{\Omega}_{n}\right) \subset C_{0}^{\infty}(E)$ such that

$$
\left[u_{n}\right]_{W^{s, p}\left(\mathbb{R}^{N}\right)}^{p}<\lambda_{p}^{s}\left(\widetilde{\Omega}_{n}\right)+\varepsilon \text { and } \int_{E}\left|u_{n}\right|^{p} \mathrm{~d} x=1 .
$$

Here the set $\widetilde{\Omega}_{n}$ is defined by

$$
\widetilde{\Omega}_{n}=\bigcup_{z \in \mathbb{Z}_{n}^{N}}(\Omega+z)=\left[-n-\frac{1}{2}, n+\frac{1}{2}\right]^{N} \backslash \bigcup_{z \in \mathbb{Z}_{n}^{N}}(F+z) .
$$

On the other hand, by Corollary 3.3 we have

$$
\begin{aligned}
\left\|u_{n}\right\|_{\mathcal{X}_{0}^{s, p}(E)}^{p} & \geq \Lambda_{p}^{s}(E) \int_{E}\left|u_{n}\right|^{p} \mathrm{~d} x \\
& =\frac{\left(\lambda_{p}^{1}(E)\right)^{s}}{p s(1-s)} \int_{E}\left|u_{n}\right|^{p} \mathrm{~d} x=\frac{\left(\lambda_{p}^{1}(E)\right)^{s}}{p s(1-s)}:=\frac{1}{C} .
\end{aligned}
$$

where we also used (A.4), to infer that $\lambda_{p}^{1}(E)>0$. By Lemma A.1, we have that $\lambda_{p}^{s}\left(\Omega_{n}\right)$ converges to 0 for $s p<1$, so that

$$
\liminf _{n \rightarrow \infty}\left\|u_{n}\right\|_{\mathcal{X}_{0}^{s, p}(E)}^{p} \geq \frac{1}{C} \text { and } \limsup _{n \rightarrow \infty}\left[u_{n}\right]_{W^{s, p}\left(\mathbb{R}^{N}\right)}^{p} \leq \varepsilon .
$$

Since $\varepsilon>0$ is arbitrary, we obtain

$$
\lim _{n \rightarrow \infty} \frac{\left\|u_{n}\right\|_{\mathcal{D}_{0}^{s, p}(E)}^{p}}{\left\|u_{n}\right\|_{\mathcal{X}_{0}^{s, p}(E)}^{p}}=0, \quad \text { for } 1<p<\infty \text { and } s<\frac{1}{p} .
$$

${ }^{2}$ In dimension $N=1$, we simply take $E=\mathbb{R} \backslash \mathbb{Z}$. 


\subsection{Convex sets}

We now prove the converse of (4.1), under suitable assumptions on $\Omega$. We start with the case of a convex set. The case $\Omega=\mathbb{R}^{N}$ is simpler and instructive; thus, we give a separate statement. The proof can be found, for example, in [28, Lemma 35.2]. We reproduce it, for the reader's convenience. We also single out an explicit determination of the constant.

Proposition 4.5 (Comparison of norms II: $\mathbb{R}^{N}$ ) Let $1<p<\infty$ and $0<s<1$. For every $u \in C_{0}^{\infty}\left(\mathbb{R}^{N}\right)$ we have

$$
\|u\|_{\mathcal{X}_{0}^{s, p}\left(\mathbb{R}^{N}\right)}^{p} \leq(N(N+1))^{p} \frac{2^{p}}{N \omega_{N}}\|u\|_{\mathcal{D}_{0}^{s, p}\left(\mathbb{R}^{N}\right)}^{p} .
$$

In particular, we have that $\mathcal{D}_{0}^{s, p}\left(\mathbb{R}^{N}\right)=\mathcal{X}_{0}^{s, p}\left(\mathbb{R}^{N}\right)$.

Proof Let $u \in C_{0}^{\infty}\left(\mathbb{R}^{N}\right)$, we set

$$
U(h)=\left(\int_{\mathbb{R}^{N}}|u(x+h)-u(x)|^{p} \mathrm{~d} x\right)^{\frac{1}{p}}, \quad h \in \mathbb{R}^{N},
$$

and observe that by construction

$$
\int_{\mathbb{R}^{N}} \frac{U(h)^{p}}{|h|^{N+s p}} \mathrm{~d} h=[u]_{W^{s, p}\left(\mathbb{R}^{N}\right)}^{p} .
$$

We also define

$$
\bar{U}(\varrho)=\frac{1}{N \omega_{N} \varrho^{N-1}} \int_{\left\{h \in \mathbb{R}^{N}:|h|=\varrho\right\}} U d \mathcal{H}^{N-1}, \quad \varrho>0 ;
$$

thus, by Jensen's inequality we have

$$
\begin{aligned}
\int_{0}^{+\infty}\left(\frac{\bar{U}}{\varrho^{s}}\right)^{p} \frac{d \varrho}{\varrho} & \leq \frac{1}{N \omega_{N}} \int_{0}^{+\infty}\left(\int_{\left\{h \in \mathbb{R}^{N}:|h|=\varrho\right\}} U^{p} d \mathcal{H}^{N-1}\right) \frac{d \varrho}{\varrho^{N+s p}} \\
& =\frac{1}{N \omega_{N}} \int_{\mathbb{R}^{N}} \frac{U(h)^{p}}{|h|^{N+s p}} \mathrm{~d} h=\frac{1}{N \omega_{N}}[u]_{W^{s, p}\left(\mathbb{R}^{N}\right)}^{p}
\end{aligned}
$$

We now take the compactly supported Lipschitz function

$$
\psi(x)=\frac{N+1}{\omega_{N}}(1-|x|)_{+},
$$

where $(\cdot)_{+}$stands for the positive part. Observe that $\psi$ has unit $L^{1}$ norm, by construction. We then define

$$
\psi_{t}(x)=\frac{1}{t^{N}} \psi\left(\frac{x}{t}\right), \quad \text { for } t>0
$$

By observing that $\psi_{t} * u \in C_{0}^{\infty}\left(\mathbb{R}^{N}\right)$, from the definition of the $K$-functional, we get

$$
K\left(t, u, L^{p}\left(\mathbb{R}^{N}\right), \mathcal{D}_{0}^{1, p}\left(\mathbb{R}^{N}\right)\right) \leq\left\|u-\psi_{t} * u\right\|_{L^{p}\left(\mathbb{R}^{N}\right)}+t\left\|\nabla \psi_{t} * u\right\|_{L^{p}\left(\mathbb{R}^{N}\right)} .
$$


We estimate the two norms in the right-hand side separately: for the first one, by Minkowski inequality and Fubini Theorem we get

$$
\begin{aligned}
\left\|u-\psi_{t} * u\right\|_{L^{p}\left(\mathbb{R}^{N}\right)} & =\left\|\int_{\mathbb{R}^{N}}[u(\cdot)-u(\cdot-y)] \psi_{t}(y) \mathrm{d} y\right\|_{L^{p}\left(\mathbb{R}^{N}\right)} \\
& \leq \int_{\mathbb{R}^{N}}\left(\int_{\mathbb{R}^{N}}|u(x)-u(x-y)|^{p} \mathrm{~d} x\right)^{\frac{1}{p}} \psi_{t}(y) \mathrm{d} y \\
& =\int_{\mathbb{R}^{N}} U(-y) \psi_{t}(y) \mathrm{d} y \leq \frac{N+1}{\omega_{N} t^{N}} \int_{B_{t}(0)} U(-y) \mathrm{d} y \\
& =\frac{N(N+1)}{t^{N}} \int_{0}^{t} \bar{U} \varrho^{N-1} d \varrho \leq \frac{N(N+1)}{t} \int_{0}^{t} \bar{U} d \varrho .
\end{aligned}
$$

For the norm of the gradient, we first observe that

$$
\int_{\mathbb{R}^{N}} \nabla \psi_{t}(y) \mathrm{d} y=0
$$

thus, we can write

$$
\nabla \psi_{t} * u=\left(\nabla \psi_{t}\right) * u=\int_{\mathbb{R}^{N}} \nabla \psi_{t}(y)[u(x-y)-u(x)] \mathrm{d} y .
$$

Consequently, by Minkowski inequality we get

$$
\begin{aligned}
\left\|\nabla \psi_{t} * u\right\|_{L^{p}\left(\mathbb{R}^{N}\right)} & =\left\|\int_{\mathbb{R}^{N}} \nabla \psi_{t}(y)[u(\cdot-y)-u(\cdot)] \mathrm{d} y\right\|_{L^{p}\left(\mathbb{R}^{N}\right)} \\
& \leq \int_{\mathbb{R}^{N}}\left(\int_{\mathbb{R}^{N}}|u(x-y)-u(x)|^{p} \mathrm{~d} x\right)^{\frac{1}{p}}\left|\nabla \psi_{t}(y)\right| \mathrm{d} y \\
& \leq \frac{N+1}{\omega_{N} t^{N+1}} \int_{B_{t}(0)} U(-y) \mathrm{d} y \leq \frac{N(N+1)}{t^{2}} \int_{0}^{t} \bar{U} d \varrho .
\end{aligned}
$$

In conclusion, we obtained for every $t>0$

$$
K\left(t, u, L^{p}\left(\mathbb{R}^{N}\right), \mathcal{D}_{0}^{1, p}\left(\mathbb{R}^{N}\right)\right) \leq \frac{2 N(N+1)}{t} \int_{0}^{t} \bar{U} d \varrho .
$$

If we integrate on $(0, T)$, the previous estimate gives

$$
\int_{0}^{T}\left(\frac{K\left(t, u, L^{p}\left(\mathbb{R}^{N}\right), \mathcal{D}_{0}^{1, p}\left(\mathbb{R}^{N}\right)\right)}{t^{s}}\right)^{p} \frac{\mathrm{d} t}{t} \leq(2 N(N+1))^{p} \int_{0}^{T}\left(\int_{0}^{t} \bar{U} d \varrho\right)^{p} t^{-p-s p} \frac{\mathrm{d} t}{t} .
$$

If we now use Lemma B.1 with $\alpha=p+s p$ for the function

$$
t \mapsto \int_{0}^{t} \bar{U} d \varrho
$$

we get

$$
\begin{aligned}
\int_{0}^{T}\left(\frac{K\left(t, u, L^{p}(\Omega), \mathcal{D}_{0}^{1, p}(\Omega)\right)}{t^{s}}\right)^{p} \frac{\mathrm{d} t}{t} & \leq\left(\frac{2 N(N+1)}{s+1}\right)^{p} \int_{0}^{T}\left(\frac{\bar{U}}{t^{s}}\right)^{p} \frac{\mathrm{d} t}{t} \\
& \leq\left(\frac{2 N(N+1)}{s+1}\right)^{p} \frac{1}{N \omega_{N}}[u]_{W^{s, p}\left(\mathbb{R}^{N}\right)}^{p},
\end{aligned}
$$

where we used (4.4) in the second inequality. By letting $T$ going to $+\infty$, we get the desired estimate. 
Remark 4.6 (Extensions by zero in $\mathcal{X}_{0}^{s, p}(\Omega) \ldots$ reprise) We take the set $E \subset \mathbb{R}^{N}$ and the sequence $\left\{u_{n}\right\}_{n \in \mathbb{N}} \subset C_{0}^{\infty}(E)$ as in Example 4.4. We have seen that

$$
\lim _{n \rightarrow \infty} \frac{\left\|u_{n}\right\|_{\mathcal{D}_{0}^{s, p}(E)}}{\left\|u_{n}\right\|_{\mathcal{X}_{0}^{s, p}(E)}}=0, \quad \text { for } s<\frac{1}{p} .
$$

By observing that

$$
\left\|u_{n}\right\|_{\mathcal{D}_{0}^{s, p}(E)}=\left\|u_{n}\right\|_{\mathcal{D}_{0}^{s, p}\left(\mathbb{R}^{N}\right)},
$$

and using Proposition 4.5, we obtain

$$
\lim _{n \rightarrow \infty} \frac{\left\|u_{n}\right\|_{\mathcal{X}_{0}^{s, p}\left(\mathbb{R}^{N}\right)}}{\left\|u_{n}\right\|_{\mathcal{X}_{0}^{s, p}(E)}}=0,
$$

as well, still for $s p<1$. This shows that the "extension by zero" operator

$$
\mathcal{T}_{0}: \mathcal{X}_{0}^{s, p}(E) \rightarrow \mathcal{X}_{0}^{s, p}\left(\mathbb{R}^{N}\right),
$$

is not an isometry and, even worse, the two norms

$$
\|\cdot\|_{\mathcal{X}_{0}^{s, p}(E)} \text { and }\|\cdot\|_{\mathcal{X}_{0}^{s, p}\left(\mathbb{R}^{N}\right)},
$$

are not equivalent on $C_{0}^{\infty}(E)$. This is in contrast with the case of $L^{p}(E), \mathcal{D}_{0}^{1, p}(E)$ and $\mathcal{D}_{0}^{s, p}(E)$.

We denote by

$$
R_{\Omega}=\sup _{x \in \Omega} \operatorname{dist}(x, \partial \Omega),
$$

the inradius of an open set $\Omega \subset \mathbb{R}^{N}$. This is the radius of the largest open ball inscribed in $\Omega$. We introduce the eccentricity of an open bounded set $\Omega \subset \mathbb{R}^{N}$, defined by

$$
\mathcal{E}(\Omega)=\frac{\operatorname{diam}(\Omega)}{2 R_{\Omega}} .
$$

Observe that this is a scaling invariant quantity. By generalizing the construction used in $[9$, Lemma A.6] for a ball, we have the following.

Theorem 4.7 (Comparison of norms II: bounded convex sets) Let $1<p<\infty$ and $0<s<1$. If $\Omega \subset \mathbb{R}^{N}$ is an open bounded convex set, then for every $u \in C_{0}^{\infty}(\Omega)$ we have

$$
\|u\|_{\mathcal{X}_{0}^{s, p}(\Omega)}^{p} \leq C\|u\|_{\mathcal{D}_{0}^{s, p}(\Omega)}^{p},
$$

for a constant $C=C(N, p, \mathcal{E}(\Omega))>0$, which blows up as $\mathcal{E}(\Omega) \rightarrow+\infty$. In particular, we have $\mathcal{X}_{0}^{s, p}(\Omega)=\mathcal{D}_{0}^{s, p}(\Omega)$.

Proof The proof runs similarly to that of Proposition 4.5 for $\mathbb{R}^{N}$, but now we have to pay attention to boundary issues. Indeed, the function $\psi_{t} * u$ is not supported in $\Omega$, unless $t$ is sufficiently small, depending on $u$ itself. In order to avoid this, we need to perform a controlled scaling of the function. By keeping the same notation as in the proof of Proposition 4.5, we need the following modification: we take a point $x_{0} \in \Omega$ such that

$$
\operatorname{dist}\left(x_{0}, \partial \Omega\right)=R_{\Omega} \text {. }
$$


Without the loss of generality, we can assume that $x_{0}=0$. Then we define the rescaled function

$$
u_{t}=u\left(\frac{R_{\Omega}}{R_{\Omega}-t} x\right), \quad 0<t<\frac{R_{\Omega}}{2} .
$$

We observe that

$$
\operatorname{support}\left(u_{t}\right)=\frac{R_{\Omega}-t}{R_{\Omega}} \Omega,
$$

and by Lemma C.1, we have

$$
\operatorname{dist}\left(\frac{R_{\Omega}-t}{R_{\Omega}} \Omega, \partial \Omega\right) \geq\left(1-\frac{R_{\Omega}-t}{R_{\Omega}}\right) R_{\Omega}=t .
$$

This implies that

$$
\psi_{t} * u_{t} \in C_{0}^{\infty}(\Omega), \quad \text { for every } 0<t<\frac{R_{\Omega}}{2} .
$$

We can now estimate the $K$-functional by using the choice $v=\psi_{t} * u_{t}$, that is

$$
\begin{aligned}
K\left(t, u, L^{p}(\Omega), \mathcal{D}_{0}^{1, p}(\Omega)\right) \leq & \left\|u-\psi_{t} * u_{t}\right\|_{L^{p}(\Omega)} \\
& +t\left\|\nabla \psi_{t} * u_{t}\right\|_{L^{p}(\Omega)}, \quad \text { for every } 0<t<\frac{R_{\Omega}}{2} .
\end{aligned}
$$

Let us set

$$
\Omega_{t}=\left\{x \in \mathbb{R}^{N}: \operatorname{dist}(x, \Omega)<2 t\right\},
$$

then we have that for every $x \in \Omega$,

$$
y \mapsto \psi_{t}(x-y) \quad \text { has support contained in } \Omega_{t} .
$$

By using this and Jensen's inequality, we obtain

$$
\left\|u-\psi_{t} * u_{t}\right\|_{L^{p}(\Omega)}^{p} \leq \int_{\Omega} \int_{\Omega_{t}}\left|u(x)-u\left(\frac{R}{R-t} y\right)\right|^{p} \frac{1}{t^{N}} \psi\left(\frac{x-y}{t}\right) \mathrm{d} y \mathrm{~d} x .
$$

Thus, by using a change of variable and Fubini theorem, we get

$$
\begin{aligned}
\int_{0}^{R_{\Omega} / 2} & \left(\frac{\left\|u-\psi_{t} * u_{t}\right\|_{L^{p}(\Omega)}}{t^{s}}\right)^{p} \frac{\mathrm{d} t}{t} \\
& \leq \int_{0}^{R_{\Omega} / 2} \int_{\Omega} \int_{\Omega_{t}} t^{-s p}\left|u(x)-u\left(\frac{R_{\Omega}}{R_{\Omega}-t} y\right)\right|^{p} \frac{1}{t^{N}} \psi\left(\frac{x-y}{t}\right) \mathrm{d} y \mathrm{~d} x \frac{\mathrm{d} t}{t} \\
& =\left(\frac{R_{\Omega}-t}{R_{\Omega}}\right)^{N} \int_{0}^{R_{\Omega} / 2} \int_{\Omega} \int_{\frac{R_{\Omega}}{R_{\Omega}-t} \Omega_{t}} t^{-s p}|u(x)-u(z)|^{p} \frac{1}{t^{N}} \psi\left(\frac{x}{t}-\frac{R_{\Omega}-t}{R_{\Omega} t} z\right) \mathrm{d} z \mathrm{~d} x \frac{\mathrm{d} t}{t} \\
& \leq \int_{\Omega} \int_{\widetilde{\Omega}}|u(x)-u(z)|^{p}\left(\int_{0}^{R_{\Omega} / 2} t^{-s p-N} \psi\left(\frac{x-z}{t}+\frac{z}{R_{\Omega}}\right) \frac{\mathrm{d} t}{t}\right) \mathrm{d} z \mathrm{~d} x,
\end{aligned}
$$

where we used that

$$
\frac{R_{\Omega}}{R_{\Omega}-t} \Omega_{t} \subset \widetilde{\Omega}:=2 \Omega_{R_{\Omega} / 2}, \quad \text { for } 0<t<\frac{R_{\Omega}}{2} .
$$

We now observe that

$$
\psi\left(\frac{x-z}{t}+\frac{z}{R_{\Omega}}\right) \neq 0 \Longleftrightarrow\left|\frac{x-z}{t}+\frac{z}{R_{\Omega}}\right|<1
$$


thus, in particular,

$$
\text { if }\left|\frac{x-z}{t}\right| \geq 1+\left|\frac{z}{R_{\Omega}}\right| \text { then } \psi\left(\frac{x-z}{t}+\frac{z}{R_{\Omega}}\right)=0
$$

i.e., for every $x \in \Omega$ and $z \in \widetilde{\Omega}$,

$$
\text { if } 0<t \leq \frac{|x-z|}{1+\frac{|z|}{R_{\Omega}}} \text { then } \psi\left(\frac{x-z}{t}+\frac{z}{R_{\Omega}}\right)=0
$$

This implies that for $x \in \Omega$ and $z \in \widetilde{\Omega}$ we get

$$
\begin{aligned}
\int_{0}^{R_{\Omega} / 2} t^{-s p-N} \psi\left(\frac{x-z}{t}+\frac{z}{R_{\Omega}}\right) \frac{\mathrm{d} t}{t} & \leq \int_{0}^{+\infty} t^{-s p-N} \psi\left(\frac{x-z}{t}+\frac{z}{R_{\Omega}}\right) \frac{\mathrm{d} t}{t} \\
& =\int_{\frac{|x-z|}{1+\frac{z \mid}{R_{\Omega}}}}^{+\infty} t^{-s p-N} \psi\left(\frac{x-z}{t}+\frac{z}{R_{\Omega}}\right) \frac{\mathrm{d} t}{t} \\
& \leq \frac{N+1}{\omega_{N}(N+s p)}\left(1+\frac{|z|}{R_{\Omega}}\right)^{N+s p}|x-z|^{-N-s p} \\
& \leq \frac{N+1}{\omega_{N}(N+s p)}\left(1+\frac{\operatorname{diam}(\widetilde{\Omega})}{R_{\Omega}}\right)^{N+s p}|x-z|^{-N-s p} .
\end{aligned}
$$

Thus, we obtain

$$
\begin{aligned}
\int_{0}^{R_{\Omega} / 2} & \left(\frac{\left\|u-\psi_{t} * u_{t}\right\|_{L^{p}(\Omega)}}{t^{s}}\right)^{p} \frac{\mathrm{d} t}{t} \\
& \leq \frac{N+1}{\omega_{N}(N+s p)}\left(1+\frac{\operatorname{diam}(\widetilde{\Omega})}{R_{\Omega}}\right)^{N+s p} \int_{\Omega} \int_{\widetilde{\Omega}} \frac{|u(x)-u(z)|^{p}}{|x-z|^{N+s p}} \mathrm{~d} x \mathrm{~d} z \\
& \leq \frac{N+1}{\omega_{N}(N+s p)}\left(1+\frac{\operatorname{diam}(\widetilde{\Omega})}{R_{\Omega}}\right)^{N+s p}\|u\|_{\mathcal{D}_{0}^{s, p}(\Omega)}^{p}
\end{aligned}
$$

Observe that by construction

$$
\operatorname{diam}(\widetilde{\Omega})=2 \operatorname{diam}\left(\Omega_{R_{\Omega} / 2}\right) \leq 2\left(\operatorname{diam}(\Omega)+2 R_{\Omega}\right) .
$$

We now need to show that

$$
\int_{0}^{R_{\Omega} / 2} t^{p}\left(\frac{\left\|\nabla \psi_{t} * u_{t}\right\|_{L^{p}(\Omega)}}{t^{s}}\right)^{p} \frac{\mathrm{d} t}{t} \leq C\|u\|_{\mathcal{D}_{0}^{s, p}(\Omega)}^{p} .
$$

We first observe that

$$
\nabla \psi_{t} * u_{t}(x)=\int_{\mathbb{R}^{N}} u\left(\frac{R_{\Omega}}{R_{\Omega}-t} y\right) \frac{1}{t^{N+1}} \nabla \psi\left(\frac{x-y}{t}\right) \mathrm{d} y,
$$

and by the divergence theorem

$$
\int_{\mathbb{R}^{N}} \frac{1}{t^{N+1}} \nabla \psi\left(\frac{x-y}{t}\right) \mathrm{d} y=0 .
$$

Thus, we obtain as well

$$
-\nabla \psi_{t} * u_{t}(x)=\int_{\mathbb{R}^{N}}\left[u\left(\frac{R_{\Omega}}{R_{\Omega}-t} x\right)-u\left(\frac{R_{\Omega}}{R_{\Omega}-t} y\right)\right] \frac{1}{t^{N+1}} \nabla \psi\left(\frac{x-y}{t}\right) \mathrm{d} y,
$$


and by Hölder's inequality

$$
\begin{aligned}
\left\|\nabla \psi_{t} * u_{t}\right\|_{L^{p}(\Omega)}^{p} \leq & \int_{\mathbb{R}^{N}}\left(\int_{\mathbb{R}^{N}}\left|u\left(\frac{R_{\Omega}}{R_{\Omega}-t} x\right)-u\left(\frac{R_{\Omega}}{R_{\Omega}-t} y\right)\right|^{p} \frac{1}{t^{N+1}}\right. \\
& \left.\left|\nabla \psi\left(\frac{x-y}{t}\right)\right| \mathrm{d} y\right) \times\left(\int_{\mathbb{R}^{N}} \frac{1}{t^{N+1}}\left|\nabla \psi\left(\frac{x-y}{t}\right)\right| \mathrm{d} y\right)^{p-1} \mathrm{~d} x \\
= & \frac{\|\nabla \psi\|_{L^{1}\left(\mathbb{R}^{N}\right)}^{p-1}}{t^{p-1}} \int_{\mathbb{R}^{N}} \int_{\mathbb{R}^{N}} \mid u\left(\frac{R_{\Omega}}{R_{\Omega}-t} x\right) \\
& -\left.u\left(\frac{R_{\Omega}}{R_{\Omega}-t} y\right)\right|^{p} \frac{1}{t^{N+1}}\left|\nabla \psi\left(\frac{x-y}{t}\right)\right| \mathrm{d} y \mathrm{~d} x \\
\leq & \frac{\|\nabla \psi\|_{L^{1}\left(\mathbb{R}^{N}\right)}^{p-1}}{t^{p-1}} \int_{\mathbb{R}^{N}} \int_{\mathbb{R}^{N}}|u(z)-u(w)|^{p} \frac{1}{t^{N+1}} \\
& \left|\nabla \psi\left(\frac{R_{\Omega}-t}{R_{\Omega} t}(z-w)\right)\right| \mathrm{d} z \mathrm{~d} w,
\end{aligned}
$$

for every $0<t<R_{\Omega} / 2$. This yields

$$
\begin{aligned}
\int_{0}^{R_{\Omega} / 2} t^{p} & \left(\frac{\left\|\nabla \psi_{t} * u_{t}\right\|_{L^{p}(\Omega)}}{t^{s}}\right)^{p} \frac{\mathrm{d} t}{t} \\
\leq & (N+1)^{p-1} \int_{0}^{R_{\Omega} / 2} t^{-s p} \int_{\mathbb{R}^{N}} \int_{\mathbb{R}^{N}}|u(z)-u(w)|^{p} \frac{1}{t^{N}} \\
& \left|\nabla \psi\left(\frac{R_{\Omega}-t}{R_{\Omega} t}(z-w)\right)\right| \mathrm{d} z \mathrm{~d} w \frac{\mathrm{d} t}{t} \\
= & (N+1)^{p-1} \int_{\mathbb{R}^{N}} \int_{\mathbb{R}^{N}}|u(z)-u(w)|^{p} \\
& \left(\int_{0}^{R_{\Omega} / 2} t^{-s p} \frac{1}{t^{N}}\left|\nabla \psi\left(\frac{R_{\Omega}-t}{R_{\Omega} t}(z-w)\right)\right| \frac{\mathrm{d} t}{t}\right) \mathrm{d} z \mathrm{~d} w .
\end{aligned}
$$

As above, we now observe that

$$
\left|\nabla \psi\left(\frac{R_{\Omega}-t}{R_{\Omega} t}(z-w)\right)\right| \neq 0 \Longleftrightarrow \frac{R_{\Omega}-t}{R_{\Omega}} \frac{|z-w|}{t}<1 ;
$$

thus, in particular, for $0<t<R_{\Omega} / 2$ we have

$$
\frac{1}{2} \frac{|z-w|}{t}>1 \Longrightarrow \nabla \psi\left(\frac{R_{\Omega}-t}{R_{\Omega} t}(z-w)\right)=0 .
$$

This implies that for $z, w \in \mathbb{R}^{N}$ we have

$$
\begin{aligned}
& \int_{0}^{R_{\Omega} / 2} t^{-s p} \frac{1}{t^{N}}\left|\nabla \psi\left(\frac{R_{\Omega}-t}{R_{\Omega} t}(z-w)\right)\right| \frac{\mathrm{d} t}{t} \\
& \quad \leq \int_{\frac{|z-w|}{2}}^{+\infty} t^{-s p} \frac{1}{t^{N}}\left|\nabla \psi\left(\frac{R_{\Omega}-t}{R_{\Omega} t}(z-w)\right)\right| \frac{\mathrm{d} t}{t} \\
& \leq \frac{(N+1) 2^{N+s p}}{\omega_{N}(N+s p)}|z-w|^{-N-s p} .
\end{aligned}
$$


By inserting this estimate in (4.9), we now get (4.8). This and (4.7) then give

$$
\int_{0}^{\frac{R_{\Omega}}{2}}\left(\frac{K\left(t, u, L^{p}(\Omega), \mathcal{D}_{0}^{1, p}(\Omega)\right)}{t^{s}}\right)^{p} \frac{\mathrm{d} t}{t} \leq C\|u\|_{\mathcal{D}_{0}^{s, p}(\Omega)}^{p},
$$

for a constant $C=C(N, p, \mathcal{E}(\Omega))$.

We are left with estimating the integral of the $K$-functional on $\left(R_{\Omega} / 2,+\infty\right)$ : for this, we can use the trivial decomposition

$$
u=(u-0)+0,
$$

which gives

$$
\begin{aligned}
\int_{\frac{R_{\Omega}}{2}}^{+\infty}\left(\frac{K\left(t, u, L^{p}(\Omega), \mathcal{D}_{0}^{1, p}(\Omega)\right)}{t^{s}}\right)^{p} \frac{\mathrm{d} t}{t} & \leq \int_{\frac{R_{\Omega}}{2}}^{+\infty} \frac{\|u\|_{L^{p}(\Omega)}^{p}}{t^{s p}} \frac{\mathrm{d} t}{t} \\
& =\frac{\|u\|_{L^{p}(\Omega)}^{p}}{s p}\left(\frac{R_{\Omega}}{2}\right)^{-s p} \\
& \leq \frac{2^{s p}}{s p}\|u\|_{\mathcal{D}_{0}^{s, p}(\Omega)}^{p}\left(\frac{1}{\lambda_{p}^{s}(\Omega) R_{\Omega}^{s p}}\right),
\end{aligned}
$$

where we used the Poincaré inequality for $\mathcal{D}_{0}^{s, p}(\Omega)$. By recalling that for a convex set with finite inradius, we have (see [8, Corollary 5.1])

$$
\lambda_{p}^{s}(\Omega) R_{\Omega}^{s p} \geq \frac{\mathcal{C}}{s(1-s)},
$$

for a constant $\mathcal{C}=\mathcal{C}(N, p)>0$, we finally obtain

$$
\int_{\frac{R_{\Omega}}{2}}^{+\infty}\left(\frac{K\left(t, u, L^{p}(\Omega), \mathcal{D}_{0}^{1, p}(\Omega)\right)}{t^{s}}\right)^{p} \frac{\mathrm{d} t}{t} \leq \frac{2^{s p}}{p}\|u\|_{\mathcal{D}_{0}^{s, p}(\Omega)}^{p}\left(\frac{1-s}{\mathcal{C}}\right) .
$$

By using this in conjunction with (4.10), we finally conclude the proof.

For general unbounded convex sets, the previous proof does not work anymore. However, for convex cones the result still holds. We say that a convex set $\Omega \subset \mathbb{R}^{N}$ is a convex cone centered at $x_{0} \in \mathbb{R}^{N}$ if for every $x \in \Omega$ and $\tau>0$, we have

$$
x_{0}+\tau\left(x-x_{0}\right) \in \Omega \text {. }
$$

Then we have the following

Corollary 4.8 (Comparison of norms II: convex cones) Let $1<p<\infty$ and $0<s<1$. If $\Omega \subset \mathbb{R}^{N}$ is an open convex cone centered at $x_{0} \in \mathbb{R}^{N}$, then for every $u \in C_{0}^{\infty}(\Omega)$ we have

$$
\|u\|_{\mathcal{X}_{0}^{s, p}(\Omega)}^{p} \leq C\|u\|_{\mathcal{D}_{0}^{s, p}(\Omega)}^{p},
$$

for a constant $C=C\left(N, p, \mathcal{E}\left(\Omega \cap B_{1}\left(x_{0}\right)\right)\right)>0$. In particular, we have $\mathcal{X}_{0}^{s, p}(\Omega)=$ $\mathcal{D}_{0}^{s, p}(\Omega)$.

Proof We assume for simplicity that $x_{0}=0$ and take $u \in C_{0}^{\infty}(\Omega)$. Since $u$ has compact support, we have that $u \in C_{0}^{\infty}\left(\Omega \cap B_{R}(0)\right)$, for $R$ large enough. From Theorem 4.7, we know that

$$
\|u\|_{\mathcal{X}_{0}^{s, p}\left(\Omega \cap B_{R}(0)\right)}^{p} \leq C\|u\|_{\mathcal{D}_{0}^{s, p}\left(\Omega \cap B_{R}(0)\right)}=C\|u\|_{\mathcal{D}_{0}^{s, p}(\Omega)} .
$$


We recall that the constant $C$ depends on the eccentricity of $\Omega \cap B_{R}(0)$. However, since $\Omega$ is a cone, we easily get

$$
\mathcal{E}\left(\Omega \cap B_{R}(0)\right)=\mathcal{E}\left(\Omega \cap B_{1}(0)\right), \quad \text { for every } R>0,
$$

i.e., the constant $C$ is independent of $R$. Finally, by observing that

$$
\|u\|_{\mathcal{X}_{0}^{s, p}(\Omega)}^{p} \leq\|u\|_{\mathcal{X}_{0}^{s, p}\left(\Omega \cap B_{R}(0)\right)}^{p},
$$

we get the desired conclusion.

Remark 4.9 (Rotationally symmetric cones) Observe that if $\Omega$ is the rotationally symmetric convex cone

$\Omega=\left\{x \in \mathbb{R}^{N}:\left\langle x-x_{0}, \omega\right\rangle>\beta\left|x-x_{0}\right|\right\}, \quad$ for some $0 \leq \beta<1, x_{0} \in \mathbb{R}^{N}$ and $\omega \in \mathbb{S}^{N-1}$, we have

$$
\mathcal{E}\left(\Omega \cap B_{1}(0)\right)=\frac{1}{2} \max \left\{2 \sqrt{1-\beta^{2}}, 1\right\}\left(1+\frac{1}{\sqrt{1-\beta^{2}}}\right),
$$

by elementary geometric considerations.

In particular, when $\Omega$ is a half-space (i.e., when $\beta=0$ ), then we have $\mathcal{E}\left(\Omega \cap B_{1}(0)\right)=2$.

\subsection{Lipschitz sets and beyond}

In this section, we show that the norms of $\mathcal{X}_{0}^{s, p}$ and $\mathcal{D}_{0}^{s, p}$ are equivalent on open bounded Lipschitz sets. We also make some comments on more general sets, see Remark 4.11.

By generalizing the idea of [22, Theorem 11.6] (see also [6, Theorem 2.1]) for $p=2$ and smooth sets, we can rely on the powerful extension theorem for Sobolev functions proved by Stein and obtain the following

Theorem 4.10 (Comparison of norms II: Lipschitz sets) Let $1<p<\infty$ and $0<s<1$. Let $\Omega \subset \mathbb{R}^{N}$ be an open bounded set, with Lipschitz, boundary. Then for every $u \in C_{0}^{\infty}(\Omega)$ we have

$$
\|u\|_{\mathcal{X}_{0}^{s, p}(\Omega)}^{p} \leq C_{1}\|u\|_{\mathcal{D}_{0}^{s, p}(\Omega)}^{p},
$$

for a constant $C_{1}>0$ depending on $N, p, \operatorname{diam}(\Omega)$ and the Lipschitz constant of $\partial \Omega$. In particular, we have $\mathcal{X}_{0}^{s, p}(\Omega)=\mathcal{D}_{0}^{s, p}(\Omega)$ in this case as well.

Proof We take an open ball $B \subset \mathbb{R}^{N}$ with radius $\operatorname{diam}(\Omega)$ and such that $\Omega \Subset B$. We then take a linear and continuous extension operator

$$
\mathcal{T}: W^{1, p}(B \backslash \bar{\Omega}) \rightarrow W^{1, p}(B),
$$

such that

$$
\left\{\begin{array}{c}
\|\mathcal{T}(u)\|_{L^{p}(B)} \leq \mathfrak{e}_{\Omega}\|u\|_{L^{p}(B \backslash \bar{\Omega})}, \\
\|\nabla \mathcal{T}(u)\|_{L^{p}(B)} \leq \mathfrak{e}_{\Omega}\|u\|_{W^{1, p}(B \backslash \bar{\Omega})},
\end{array}\right.
$$

where $\mathfrak{e}_{\Omega}>0$ depends on $N, p, \operatorname{diam}(\Omega)$ and the Lipschitz constant of $\partial \Omega$. We observe that such an operator exists, thanks to the fact that $\Omega$ has a Lipschitz boundary, see [27, Theorem 5, p. 181]. We also observe that the first estimate in (4.11) is not explicitly stated by Stein, but it can be extrapolated by having a closer look at the proof, see [27, p. 192]. 
For every $v \in C_{0}^{\infty}(B)$, we define the operator

$$
\mathcal{R}(v)=v-\mathcal{T}\left(v \cdot 1_{B \backslash \bar{\Omega}}\right),
$$

and observe that

$$
\mathcal{R}(v) \equiv 0 \text { in } B \backslash \bar{\Omega} \text { and } \mathcal{R}(v) \in W^{1, p}(B) .
$$

Since $\Omega$ has continuous boundary, this implies that $\mathcal{R}(v) \in \mathcal{D}_{0}^{1, p}(\Omega)$, see Remark 2.1. We now fix $u \in C_{0}^{\infty}(B)$, for every $v \in C_{0}^{\infty}(B)$ and every $t, \varepsilon>0$, we take $\varphi_{\varepsilon, t} \in C_{0}^{\infty}(\Omega)$ such that

$$
\left(\lambda_{p}^{1}(\Omega)\right)^{\frac{1}{p}}\left\|\varphi_{\varepsilon}-\mathcal{R}(v)\right\|_{L^{p}(\Omega)} \leq\left\|\nabla \varphi_{\varepsilon, t}-\nabla \mathcal{R}(v)\right\|_{L^{p}(\Omega)}<\varepsilon\left(t+\left(\lambda_{p}^{1}(\Omega)\right)^{-\frac{1}{p}}\right)^{-1} .
$$

This is possible, thanks to the definition of $\mathcal{D}_{0}^{1, p}(\Omega)$. Then for $t>0$ we can estimate the relevant $K$-functional as follows

$$
\begin{aligned}
K\left(t, \mathcal{R}(u), L^{p}(\Omega), \mathcal{D}_{0}^{1, p}(\Omega)\right) & \leq\left\|\mathcal{R}(u)-\varphi_{\varepsilon, t}\right\|_{L^{p}(\Omega)}+t\left\|\nabla \varphi_{\varepsilon, t}\right\|_{L^{p}(\Omega)} \\
& \leq\|\mathcal{R}(u)-\mathcal{R}(v)\|_{L^{p}(\Omega)}+\left\|\varphi_{\varepsilon, t}-\mathcal{R}(v)\right\|_{L^{p}(\Omega)} \\
& +t\|\nabla \mathcal{R}(v)\|_{L^{p}(\Omega)}+t\left\|\nabla \varphi_{\varepsilon, t}-\nabla \mathcal{R}(v)\right\|_{L^{p}(\Omega)} \\
& \leq\|\mathcal{R}(u-v)\|_{L^{p}(\Omega)}+t\|\nabla \mathcal{R}(v)\|_{L^{p}(\Omega)} \\
& +\left(t+\left(\lambda_{p}^{1}(\Omega)\right)^{-\frac{1}{p}}\right)\left\|\nabla \varphi_{\varepsilon, t}-\nabla \mathcal{R}(v)\right\|_{L^{p}(\Omega)} \\
& \leq\|u-v\|_{L^{p}(\Omega)}+\left\|\mathcal{T}\left((u-v) \cdot 1_{B \backslash \bar{\Omega}^{2}}\right)\right\|_{L^{p}(\Omega)} \\
& +t\left(\|\nabla v\|_{L^{p}(\Omega)}+\left\|\nabla \mathcal{T}\left(v \cdot 1_{B \backslash \bar{\Omega}}\right)\right\|_{L^{p}(\Omega)}\right)+\varepsilon .
\end{aligned}
$$

By applying (4.11) and using that

$\left\|\nabla \mathcal{T}\left(v \cdot 1_{B \backslash \bar{\Omega}}\right)\right\|_{L^{p}(B)} \leq \mathfrak{e}_{\Omega}\left\|v \cdot 1_{B \backslash \bar{\Omega}}\right\|_{W^{1, p}(B \backslash \bar{\Omega})}=\mathfrak{e}_{\Omega}\|v\|_{W^{1, p}(B \backslash \bar{\Omega})} \leq \mathfrak{e}_{\Omega}\|v\|_{W^{1, p}(B)}$, we then get

$$
K\left(t, \mathcal{R}(u), L^{p}(\Omega), \mathcal{D}_{0}^{1, p}(\Omega)\right) \leq\left(1+\mathfrak{e}_{\Omega}\right)\|u-v\|_{L^{p}(B)}+t\left(\|\nabla v\|_{L^{p}(B)}+\mathfrak{e}_{\Omega}\|v\|_{W^{1, p}(B)}\right)+\varepsilon .
$$

We now use that

$$
\|v\|_{W^{1, p}(B)}=\left(\|v\|_{L^{p}(B)}^{p}+\|\nabla v\|_{L^{p}(B)}^{p}\right)^{\frac{1}{p}} \leq\|\nabla v\|_{L^{p}(B)}\left(1+\frac{1}{\lambda_{p}^{1}(B)}\right)^{\frac{1}{p}},
$$

thanks to Poincaré inequality. By spending this information in the previous estimate and using the arbitrariness of $\varepsilon$, we get

$$
\begin{aligned}
K\left(t, \mathcal{R}(u), L^{p}(\Omega), \mathcal{D}_{0}^{1, p}(\Omega)\right) & \leq\left(1+\mathfrak{e}_{\Omega}\right)\|u-v\|_{L^{p}(B)} \\
& +t\left(1+\mathfrak{e}_{\Omega}\left(1+\frac{1}{\lambda_{p}^{1}(B)}\right)^{\frac{1}{p}}\right)\|\nabla v\|_{L^{p}(B)} .
\end{aligned}
$$


We set for simplicity

$$
\gamma_{\Omega}=1+\mathfrak{e}_{\Omega}\left(1+\frac{1}{\lambda_{p}^{1}(B)}\right)^{\frac{1}{p}}
$$

then by taking the infimum over $v \in C_{0}^{\infty}(B)$

$$
K\left(t, \mathcal{R}(u), L^{p}(\Omega), \mathcal{D}_{0}^{1, p}(\Omega)\right) \leq \gamma_{\Omega} K\left(t, u, L^{p}(B), \mathcal{D}_{0}^{1, p}(B)\right) .
$$

As usual, we integrate in $t$, so to get

$$
\|\mathcal{R}(u)\|_{\mathcal{X}_{0}^{s, p}(\Omega)}^{p} \leq \gamma_{\Omega}^{p}\|u\|_{\mathcal{X}_{0}^{s, p}(B)}^{p}, \quad \text { for } u \in C_{0}^{\infty}(B) .
$$

We now observe that if $u \in C_{0}^{\infty}(\Omega)$, then we have $\mathcal{R}(u)=u$. Thus, from (4.12) and Theorem 4.7 for the convex set $B$, we get

$$
\|u\|_{\mathcal{X}_{0}^{s, p}(\Omega)}^{p} \leq C \gamma_{\Omega}^{p}\|u\|_{\mathcal{D}_{0}^{s, p}\left(\mathbb{R}^{N}\right)}^{p}=C \gamma_{\Omega}^{p}\|u\|_{\mathcal{D}_{0}^{s, p}(\Omega)}^{p}, \quad \text { for every } u \in C_{0}^{\infty}(\Omega),
$$

where $C$ only depends on $N$ and $p$. This concludes the proof.

Remark 4.11 (More general sets) It is not difficult to see that the previous proof works (and thus $\mathcal{X}_{0}^{s, p}(\Omega)$ and $\mathcal{D}_{0}^{s, p}(\Omega)$ are equivalent), whenever the set $\Omega$ is such that there exists a linear and continuous extension operator

$$
\mathcal{T}: W^{1, p}(B \backslash \bar{\Omega}) \rightarrow W^{1, p}(B),
$$

such that (4.11) holds. Observe that there is a vicious subtlety here: the first condition in (4.11) is vital and, in general, it may fail to hold for an extension operator. For example, there is a beautiful extension result by Jones [21, Theorem 1], which is valid for very irregular domains (possibly having a fractal boundary): however, the construction given by Jones does not assure that the first estimate in (4.11) holds true, see the statement of [21, Lemma 3.2].

In order to complement the discussion of Remarks 3.4 and 4.6 on "extensions by zero" in $\mathcal{X}_{0}^{s, p}$, we explicitly state the following consequence of Proposition 4.1 and Theorem 4.10.

Corollary 4.12 Let $1<p<\infty$ and $0<s<1$. Let $\Omega \subset \mathbb{R}^{N}$ be an open bounded set, with Lipschitz boundary. Then for every $u \in C_{0}^{\infty}(\Omega)$, we have

$$
\|u\|_{\mathcal{X}_{0}^{s, p}(\Omega)}^{p} \leq 2^{p(1-s)} N \omega_{N} C_{1}\|u\|_{\mathcal{X}_{0}^{s, p}\left(\mathbb{R}^{N}\right)}^{p},
$$

where $C_{1}>0$ is the same constant as in Theorem 4.10 .

\section{Capacities}

Let $1<p<N$, we recall that for every compact set $F \subset \mathbb{R}^{N}$, its $p$-capacity is defined by

$$
\operatorname{cap}_{p}(F)=\inf _{u \in C_{0}^{\infty}\left(\mathbb{R}^{N}\right)}\left\{\int_{\mathbb{R}^{N}}|\nabla u|^{p} \mathrm{~d} x: u \geq 0 \text { and } u \geq 1_{F}\right\},
$$

see [17, Chapter 4, Section 7]. 
Similarly, given $1<p<\infty$ and $0<s<1$ such that ${ }^{3} s p<N$, we define the $(s, p)$ capacity of $F$ through

$$
\operatorname{cap}_{s, p}(F)=\inf _{u \in C_{0}^{\infty}\left(\mathbb{R}^{N}\right)}\left\{[u]_{W^{s, p}\left(\mathbb{R}^{N}\right)}^{p}: u \geq 0 \text { and } u \geq 1_{F}\right\}
$$

and the interpolation $(s, p)$-capacity of $F$ by

$$
\text { int } \operatorname{cap}_{s, p}(F)=\inf _{u \in C_{0}^{\infty}\left(\mathbb{R}^{N}\right)}\left\{\|u\|_{\mathcal{X}_{0}^{s, p}\left(\mathbb{R}^{N}\right)}^{p}: u \geq 0 \text { and } u \geq 1_{F}\right\} \text {. }
$$

As a straightforward consequence of Propositions 4.1 and 4.5, we have the following

Corollary 5.1 (Comparison of capacities) Let $1<p<\infty$ and $0<s<1$ be such that s $p<N$. Let $F \subset \mathbb{R}^{N}$ be a compact set, then we have

$$
\frac{1}{C} \operatorname{cap}_{s, p}(F) \leq \operatorname{int} \operatorname{cap}_{s, p}(F) \leq C \operatorname{cap}_{s, p}(F),
$$

for a constant $C=C(N, p)>1$. In particular, it holds

$$
\operatorname{cap}_{s, p}(F)=0 \text { if and only if int cap } s, p(F)=0 \text {. }
$$

Proposition 5.2 Let $1<p<\infty$ and $0<s<1$ be such that $s p<N$. For every $E, F \subset \mathbb{R}^{N}$ compact sets, we have

$$
\operatorname{cap}_{s, p}(E \cup F) \leq \operatorname{cap}_{s, p}(E)+\operatorname{cap}_{s, p}(F) .
$$

Proof We fix $n \in \mathbb{N} \backslash\{0\}$ and choose two nonnegative functions $\varphi_{n}, \psi_{n} \in C_{0}^{\infty}\left(\mathbb{R}^{N}\right)$ such that

$$
\left[\varphi_{n}\right]_{W^{s, p}\left(\mathbb{R}^{N}\right)}^{p} \leq \operatorname{cap}_{s, p}(E)+\frac{1}{n}, \quad \varphi_{n} \geq 1_{E},
$$

and

$$
\left[\psi_{n}\right]_{W^{s, p}\left(\mathbb{R}^{N}\right)}^{p} \leq \operatorname{cap}_{s, p}(F)+\frac{1}{n}, \quad \psi_{n} \geq 1_{F} .
$$

We then set

$$
U_{n, \varepsilon}=\left(\max \left\{\varphi_{n}, \psi_{n}\right\}\right) * \varrho_{\varepsilon}, \quad 0<\varepsilon \ll 1,
$$

where $\left\{\varrho_{\varepsilon}\right\}_{\varepsilon>0}$ is a family of standard Friedrichs mollifiers. We observe that for every $n \in$ $\mathbb{N} \backslash\{0\}$, it holds that $U_{n, \varepsilon} \in C_{0}^{\infty}\left(\mathbb{R}^{N}\right)$. Moreover, by construction we have

$$
U_{n, \varepsilon} \geq 1_{E \cup F} .
$$

By observing that Jensen's inequality implies

$$
\left[U_{n, \varepsilon}\right]_{W^{s, p}\left(\mathbb{R}^{N}\right)} \leq\left[\max \left\{\varphi_{n}, \psi_{n}\right\}\right]_{W^{s, p}\left(\mathbb{R}^{N}\right)}^{p},
$$

we thus get

$$
\operatorname{cap}_{s, p}(E \cup F) \leq\left[U_{n, \varepsilon}\right]_{W^{s, p}\left(\mathbb{R}^{N}\right)} \leq\left[\max \left\{\varphi_{n}, \psi_{n}\right\}\right]_{W^{s, p}\left(\mathbb{R}^{N}\right)}^{p} .
$$

3 As usual, the restriction $s p<N$ is due to the scaling properties of the relevant energies. It is not difficult to see that for $s p \geq N$, both infima are identically 0 . 
By using the sub-modularity of the Sobolev-Slobodeckir seminorm (see [19, Theorem 3.2 \& Remark 3.3]), we obtain

$$
\operatorname{cap}_{s, p}(E \cup F) \leq\left[\varphi_{n}\right]_{W^{s, p}\left(\mathbb{R}^{N}\right)}^{p}+\left[\psi_{n}\right]_{W^{s, p}\left(\mathbb{R}^{N}\right)}^{p} .
$$

Finally, thanks to the choice of $\varphi_{n}$ and $\psi_{n}$, we get the desired conclusion by the arbitrariness of $n$.

In the next result, we denote by $\mathcal{H}^{\tau}$ the $\tau$-dimensional Hausdorff measure.

Proposition 5.3 Let $1<p<\infty$ and $0<s<1$ be such that $s p<N$. Let $\Omega \subset \mathbb{R}^{N}$ be an open set. We take a compact set $E \Subset \Omega$ such that

$$
\operatorname{cap}_{s, p}(E)=0 .
$$

Then we have

$$
\mathcal{H}^{\tau}(E)=0 \text { for every } \tau>N-s p
$$

and

$$
\lambda_{p}^{s}(\Omega \backslash E)=\lambda_{p}^{s}(\Omega) .
$$

Proof To prove (5.1), we can easily adapt the proof of [17, Theorem 4, p. 156], dealing with the local case.

In order to prove (5.2), we first assume $\Omega$ to be bounded. Let $\varepsilon>0$, we take $u_{\varepsilon} \in C_{0}^{\infty}(\Omega)$ such that

$$
\left\|u_{\varepsilon}\right\|_{\mathcal{D}_{0}^{s, p}(\Omega)}^{p}<(1+\varepsilon) \lambda_{p}^{s}(\Omega) \text { and } \int_{\Omega}\left|u_{\varepsilon}\right|^{p} \mathrm{~d} x=1 .
$$

We further observe that the boundedness of $\Omega$ implies that

$$
\lambda_{p}^{s}(\Omega)=\inf _{u \in C_{0}^{\infty}(\Omega)}\left\{\|u\|_{\mathcal{D}_{0}^{s, p}(\Omega)}^{p}:\|u\|_{L^{p}(\Omega)}=1\right\}=\min _{u \in \mathcal{D}_{0}^{s, p}(\Omega)}\left\{\|u\|_{\mathcal{D}_{0}^{s, p}(\Omega)}^{p}:\|u\|_{L^{p}(\Omega)}=1\right\},
$$

and that any solution $u \in \mathcal{D}_{0}^{s, p}(\Omega)$ has norm $L^{\infty}(\Omega)$ bounded by a constant $M=$ $M(N, s, p, \Omega)$, see [7, Theorem 3.3]. Thus, without the loss of generality, we can also assume that

$$
\left\|u_{\varepsilon}\right\|_{L^{\infty}(\Omega)} \leq M+1, \quad \text { for } 0<\varepsilon \ll 1 .
$$

Since $E$ has null $(s, p)$-capacity, there exists $\varphi_{\varepsilon} \in C_{0}^{\infty}(\Omega)$ such that

$$
\left[\varphi_{\varepsilon}\right]_{W^{s, p}\left(\mathbb{R}^{N}\right)}^{p}<\varepsilon, \quad \varphi_{\varepsilon} \geq 0 \text { and } \varphi_{\varepsilon} \geq 1_{E} .
$$

We set $\psi_{\varepsilon}=\varphi_{\varepsilon} /\left\|\varphi_{\varepsilon}\right\|_{L^{\infty}\left(\mathbb{R}^{N}\right)}$ and observe that $\left\|\varphi_{\varepsilon}\right\|_{L^{\infty}\left(\mathbb{R}^{N}\right)} \geq 1$. The function $u_{\varepsilon}\left(1-\psi_{\varepsilon}\right)$ is admissible for the variational problem defining $\lambda_{p}^{s}(\Omega \backslash E)$; then by using the triangle inequality, we have

$$
\begin{aligned}
\left(\lambda_{p}^{s}(\Omega \backslash E)\right)^{\frac{1}{p}} \leq \frac{\left[u_{\varepsilon}\left(1-\psi_{\varepsilon}\right)\right]_{W^{s, p}\left(\mathbb{R}^{N}\right)}}{\left\|u_{\varepsilon}\left(1-\psi_{\varepsilon}\right)\right\|_{L^{p}(\Omega \backslash E)}} \leq & \frac{\left[u_{\varepsilon}\right]_{W^{s, p}\left(\mathbb{R}^{N}\right)}\left\|1-\psi_{\varepsilon}\right\|_{L^{\infty}\left(\mathbb{R}^{N}\right)}}{\left\|u_{\varepsilon}\left(1-\psi_{\varepsilon}\right)\right\|_{L^{p}(\Omega \backslash E)}} \\
& +\frac{\left\|u_{\varepsilon}\right\|_{L^{\infty}}\left[\psi_{\varepsilon}\right]_{W^{s, p}\left(\mathbb{R}^{N}\right)}}{\left\|u_{\varepsilon}\left(1-\psi_{\varepsilon}\right)\right\|_{L^{p}(\Omega \backslash E)}} .
\end{aligned}
$$

From the first part of the proof, we know that $E$ has $N$-dimensional Lebesgue measure 0; thus, the $L^{p}$ norm over $\Omega \backslash E$ is the same as that over $\Omega$. If we now take the limit as $\varepsilon$ goes 
to 0 and use the properties of $u_{\varepsilon}$, together with ${ }^{4}$

$$
\left[\psi_{\varepsilon}\right]_{W^{s, p}\left(\mathbb{R}^{N}\right)}^{p}<\frac{\varepsilon^{p}}{\left\|\varphi_{\varepsilon}\right\|_{L^{\infty}\left(\mathbb{R}^{N}\right)}} \leq \varepsilon^{p},
$$

and

$$
\lim _{\varepsilon \rightarrow 0}\left\|1-\psi_{\varepsilon}\right\|_{L^{\infty}\left(\mathbb{R}^{N}\right)}=\lim _{\varepsilon \rightarrow 0} \sup _{\mathbb{R}^{N}}\left(1-\psi_{\varepsilon}\right) \leq 1,
$$

we get

$$
\left(\lambda_{p}^{s}(\Omega \backslash E)\right)^{\frac{1}{p}} \leq\left(\lambda_{p}^{s}(\Omega)\right)^{\frac{1}{p}} .
$$

The reverse inequality simply follows from the fact that $C_{0}^{\infty}(\Omega \backslash E) \subset C_{0}^{\infty}(\Omega)$; thus, we get the conclusion when $\Omega$ is bounded.

In order to remove the last assumption, we consider the sets $\Omega_{R}=\Omega \cap B_{R}(0)$. For $R$ large enough, this is a nonempty open bounded set and $E \Subset \Omega_{R}$ as well. We thus have

$$
\lambda_{p}^{s}\left(\Omega_{R} \backslash E\right)=\lambda_{p}^{s}\left(\Omega_{R}\right) .
$$

By taking the limit ${ }^{5}$ as $R$ goes to $+\infty$, we get the desired conclusion in the general case as well.

The previous result giving the link between the Poincaré constant and sets with null capacity does not hold true in the interpolation space $\mathcal{X}_{0}^{s, p}(\Omega)$. Indeed, we have the following result, which shows that the interpolation Poincare constant is sensitive to removing sets with null $(s, p)$-capacity.

Lemma 5.4 Let $1<p<N$ and $0<s<1$. Let $\Omega \subset \mathbb{R}^{N}$ be an open set and $E \Subset \Omega$ a compact set such that

$$
\text { int } \operatorname{cap}_{s, p}(E)=0<\operatorname{cap}_{p}(E) \text {. }
$$

Then we have

$$
\Lambda_{p}^{s}(\Omega \backslash E)>\Lambda_{p}^{s}(\Omega)
$$

Proof By Corollary 3.3, we know that

$$
p s(1-s) \Lambda_{p}^{s}(\Omega \backslash E)=\left(\lambda_{p}^{1}(\Omega \backslash E)\right)^{s} \text { and } p s(1-s) \Lambda_{p}^{s}(\Omega)=\left(\lambda_{p}^{1}(\Omega)\right)^{s} .
$$

It is now sufficient to use that $\lambda_{p}^{1}(\Omega \backslash E)>\lambda_{p}^{1}(\Omega)$, as a consequence of the fact that $E$ has positive $p$-capacity.

4 Observe that, from the first condition, we get that $\psi_{\varepsilon}$ converges to 0 strongly in $L^{p}(\Omega)$, by Sobolev inequality. Since the family $\left\{u_{\varepsilon}\right\}$ is bounded in $L^{\infty}(\Omega)$, this is enough to infer

$$
\lim _{\varepsilon \rightarrow 0} \int_{\Omega}\left|u_{\varepsilon}\right|^{p}\left|1-\psi_{\varepsilon}\right|^{p} \mathrm{~d} x=1 .
$$

5 Such a limit exists by monotonicity. 
Remark 5.5 As an explicit example of the previous situation, we can take $s p<1$ and the $(N-1)$-dimensional set

$$
F=[-a, a]^{N-1} \times\{0\} .
$$

Observe that $\operatorname{cap}_{p}(F)>0$ by [17, Theorem 4, p. 156]. On the other hand, we have

$$
\text { int } \operatorname{cap}_{s, p}(F)=0 \text {. }
$$

Indeed, we set

$$
F_{\varepsilon}=\left\{x \in \mathbb{R}^{N}: \operatorname{dist}(x, F)<\varepsilon\right\} .
$$

We then take the usual sequence of Friedrichs mollifiers $\left\{\varrho_{\varepsilon}\right\}_{\varepsilon>0} \subset C_{0}^{\infty}\left(\mathbb{R}^{N}\right)$ and define

$$
\varphi_{\varepsilon}=1_{F_{\varepsilon}} * \varrho_{\varepsilon} \in C_{0}^{\infty}\left(\mathbb{R}^{N}\right) .
$$

Observe that by construction we have

$$
\varphi_{\varepsilon} \equiv 1 \text { on } F_{\varepsilon} \text { and } \varphi_{\varepsilon} \equiv 0 \text { on } \mathbb{R}^{N} \backslash F_{2 \varepsilon} .
$$

By definition of $(s, p)$-capacity and using the interpolation estimate (4.3), we get

$$
\begin{aligned}
\operatorname{cap}_{s, p}(F) & \leq\left[\varphi_{\varepsilon}\right]_{W^{s, p}\left(\mathbb{R}^{N}\right)}^{p} \\
& \leq C\left(\int_{\mathbb{R}^{N}}\left|\varphi_{\varepsilon}\right|^{p} \mathrm{~d} x\right)^{1-s}\left(\int_{\mathbb{R}^{N}}\left|\nabla \varphi_{\varepsilon}\right|^{p} \mathrm{~d} x\right)^{s} \\
& \leq C\left(\int_{\mathbb{R}^{N}}\left|1_{F_{\varepsilon}}\right|^{p} \mathrm{~d} x\right)^{1-s}\left(\int_{\mathbb{R}^{N}}\left|1_{F_{\varepsilon}}\right|^{p} \mathrm{~d} x\right)^{s}\left(\int_{\mathbb{R}^{N}}\left|\nabla \varrho_{\varepsilon}\right| \mathrm{d} x\right)^{s p} \\
& \leq C\left|F_{\varepsilon}\right| \varepsilon^{-s p} \leq C \varepsilon^{1-s p} .
\end{aligned}
$$

We then observe that the last quantity goes to 0 as $\varepsilon$ goes to 0 , thanks to the fact that $s p<1$. By Corollary 5.1, we have

$$
\text { int } \operatorname{cap}_{s, p}(F)=\operatorname{cap}_{s, p}(F)=0 \text {. }
$$

as desired.

\section{Double-sided estimates for Poincaré constants}

We already observed that for an open set $\Omega \subset \mathbb{R}^{N}$ we have

$$
p s(1-s) \Lambda_{p}^{s}(\Omega)=\left(\lambda_{p}^{1}(\Omega)\right)^{s} .
$$

We now want to compare $\lambda_{p}^{1}$ with the sharp Poincaré constant for the embedding $\mathcal{D}_{0}^{s, p}(\Omega) \hookrightarrow$ $L^{p}(\Omega)$.

Theorem 6.1 Let $1<p<\infty$ and $0<s<1$. Let $\Omega \subset \mathbb{R}^{N}$ be an open set, then

$$
s(1-s) \lambda_{p}^{s}(\Omega) \leq \frac{2^{p(1-s)} N \omega_{N}}{p}\left(\lambda_{p}^{1}(\Omega)\right)^{s} .
$$

If in addition:

袋 Springer 
- $\Omega \subset \mathbb{R}^{N}$ is bounded with Lipschitz, boundary, then we also have the reverse inequality

$$
\frac{1}{p C_{1}}\left(\lambda_{p}^{1}(\Omega)\right)^{s} \leq s(1-s) \lambda_{p}^{s}(\Omega),
$$

where $C_{1}>0$ is the same constant as in Theorem 4.10;

- $\Omega \subset \mathbb{R}^{N}$ is convex, then we also have the reverse inequality

$$
\frac{1}{C_{2}}\left(\lambda_{p}^{1}(\Omega)\right)^{s} \leq s(1-s) \lambda_{p}^{s}(\Omega),
$$

where $C_{2}$ is the universal constant given by

$$
C_{2}=\frac{\left(\lambda_{p}^{1}\left(B_{1}(0)\right)\right)^{s}}{\mathcal{C}}
$$

and $\mathcal{C}=\mathcal{C}(N, p)>0$ is the same constant as in the Hardy inequality for $\mathcal{D}_{0}^{s, p}(\Omega)$ (see $[8$, Theorem 1.1]).

Proof The first inequality (6.1) is a direct consequence of the interpolation inequality (4.3). Indeed, by using the definition of $\lambda_{p}^{s}(\Omega)$, we obtain from this inequality

$$
s(1-s) \lambda_{p}^{s}(\Omega)\|u\|_{L^{p}(\Omega)}^{p} \leq \frac{2^{p(1-s)} N \omega_{N}}{p}\|u\|_{L^{p}(\Omega)}^{(1-s) p}\|\nabla u\|_{L^{p}(\Omega)}^{s p},
$$

for every $u \in C_{0}^{\infty}(\Omega)$. By simplifying the factor $\|u\|_{L^{p}(\Omega)}^{p}$ on both sides and taking the infimum over $C_{0}^{\infty}(\Omega)$, we get the claimed inequality.

In order to prove (6.2), for every $\varepsilon>0$ we take $\varphi \in C_{0}^{\infty}(\Omega)$ such that

$$
\frac{\|\varphi\|_{\mathcal{D}_{0}^{s, p}(\Omega)}^{p}}{\|\varphi\|_{L^{p}(\Omega)}^{p}}<\lambda_{p}^{s}(\Omega)+\varepsilon
$$

then we use Theorem 4.10 to infer

$$
\frac{1}{C_{1}} \frac{\|\varphi\|_{\mathcal{X}_{0}^{s, p}(\Omega)}^{p}}{\|\varphi\|_{L^{p}(\Omega)}^{p}}<\lambda_{p}^{s}(\Omega)+\varepsilon
$$

This in turn implies

$$
\frac{1}{C_{1}} \Lambda_{p}^{s}(\Omega) \leq \lambda_{p}^{s}(\Omega)
$$

by arbitrariness of $\varepsilon>0$. A further application of Corollary 3.3 leads to the desired conclusion.

Finally, if $\Omega \subset \mathbb{R}^{N}$ is convex, we can proceed in a different way. We first observe that we can always suppose that the inradius $R_{\Omega}$ is finite; otherwise, both $\lambda_{p}^{1}(\Omega)$ and $\lambda_{p}^{s}(\Omega)$ vanish, and there is nothing to prove. Then (6.3) comes by joining the simple estimate

$$
\lambda_{p}^{1}(\Omega) \leq \frac{\lambda_{p}^{1}\left(B_{1}(0)\right)}{R_{\Omega}^{p}},
$$

which follows from the monotonicity and scaling properties of $\lambda_{p}^{1}$, and the estimate of $[8$, Corollary 5.1], i.e.,

$$
s(1-s) \lambda_{p}^{s}(\Omega) \geq \frac{\mathcal{C}}{R_{\Omega}^{s p}}
$$


The latter is a consequence of the Hardy inequality in convex sets for $\mathcal{D}_{0}^{s, p}$.

Remark 6.2 For $p=2$, the double-sided estimate of Theorem 6.1 is contained in [12, Theorem 4.5]. The proof in [12] relies on probabilistic techniques, and the result is proved by assuming that $\Omega$ verifies a uniform exterior cone condition.

Remark 6.3 Inequality (6.2) cannot hold for a general open set $\Omega \subset \mathbb{R}^{N}$, with a constant independent of $\Omega$. Indeed, one can construct a sequence $\left\{\Omega_{n}\right\}_{n \in \mathbb{N}} \subset \mathbb{R}^{N}$ such that

$$
\lim _{n \rightarrow \infty} \frac{\left(\lambda_{p}^{1}\left(\Omega_{n}\right)\right)^{s}}{\lambda_{p}^{s}\left(\Omega_{n}\right)}=+\infty, \quad \text { for } 1<p<\infty \text { and } s<\frac{1}{p},
$$

see Lemma A.1.

Acknowledgements The first author would like to thank Yavar Kian and Antoine Lemenant for useful discussions on Stein's and Jones' extension theorems. Simon Chandler-Wilde is gratefully acknowledged for some explanations on his paper [11]. This work started during a visit of the second author to the University of Ferrara in October 2017.

\section{Appendix A. An example}

In this section, we construct a sequence of open bounded sets $\left\{\Omega_{n}\right\}_{n \in \mathbb{N}} \subset \mathbb{R}^{N}$ with rough boundaries and fixed diameter, such that we have

$$
\lim _{n \rightarrow \infty} \frac{\left(\lambda_{p}^{1}\left(\Omega_{n}\right)\right)^{s}}{\lambda_{p}^{s}\left(\Omega_{n}\right)}=+\infty, \quad \text { for } 1<p<\infty \text { and } s<\frac{1}{p} .
$$

The sets $\Omega_{n}$ are obtained by removing from an $N$-dimensional cube an increasing array of regular $(N-1)$-dimensional cracks (Fig. 1).

For $N \geq 1$, we set 6

$$
Q=\left[-\frac{1}{2}, \frac{1}{2}\right]^{N} \text { and } F=\left[-\frac{1}{4}, \frac{1}{4}\right]^{N-1} \times\{0\} .
$$

For every $n \in \mathbb{N}$, we also define

$$
\mathbb{Z}_{n}^{N}=\left\{z=\left(z_{1}, \ldots, z_{N}\right) \in \mathbb{Z}^{N}: \max \left\{\left|z_{1}\right|, \ldots,\left|z_{N}\right|\right\} \leq n\right\} .
$$

Finally, we consider the sets

$$
\Omega=Q \backslash F, \quad \widetilde{\Omega}_{n}=\bigcup_{z \in \mathbb{Z}_{n}^{N}}(\Omega+z)=\left[-n-\frac{1}{2}, n+\frac{1}{2}\right]^{N} \backslash \bigcup_{z \in \mathbb{Z}_{n}^{N}}(F+z),
$$

and

$$
E=\bigcup_{n \in \mathbb{N}} \widetilde{\Omega}_{n}=\mathbb{R}^{N} \backslash \bigcup_{z \in \mathbb{Z}^{N}}(F+z) .
$$

Then (A.1) is a consequence of the next result.

6 For $N=1$, the set $F$ simply coincides with the point $\{0\}$. 


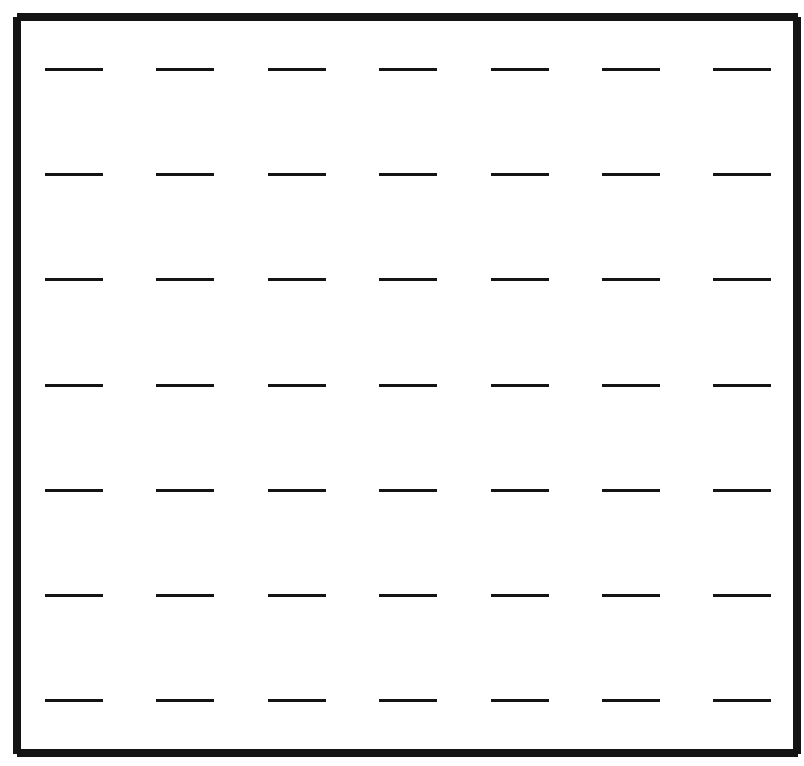

Fig. 1 The set $\Omega_{n}$ in dimension $N=2$, for $n=3$

Lemma A.1 With the notation above, for $1<p<\infty$ and $s<1 / p$ we have

$$
\lambda_{p}^{1}\left(\widetilde{\Omega}_{n}\right) \geq C=C(N, p, F)>0, \text { for every } n \in \mathbb{N},
$$

and

$$
\lim _{n \rightarrow \infty} \lambda_{p}^{s}\left(\widetilde{\Omega}_{n}\right)=0
$$

In particular, the new sequence of rescaled sets $\left\{\Omega_{n}\right\}_{n \in \mathbb{N}} \subset \mathbb{R}^{N}$ defined by

$$
\Omega_{n}=\left|\widetilde{\Omega}_{n}\right|^{-\frac{1}{N}} \widetilde{\Omega}_{n}=\left[-\frac{1}{2}, \frac{1}{2}\right]^{N} \backslash \bigcup_{z \in \mathbb{Z}_{n}^{N}} \frac{(F+z)}{2 n+1},
$$

is such that

$$
\operatorname{diam}\left(\Omega_{n}\right)=\sqrt{N}, \text { for every } n \in \mathbb{N} \text { and } \lim _{n \rightarrow \infty} \frac{\left(\lambda_{p}^{1}\left(\Omega_{n}\right)\right)^{s}}{\lambda_{p}^{s}\left(\Omega_{n}\right)}=+\infty .
$$

Proof We divide the proof in two parts, for ease of readability. Of course, it is enough to prove (A.2) and (A.3). Indeed, the last statement is a straightforward consequence of these facts and of the scaling properties of the diameter and of the Poincaré constants.

Proof of (A.2). For $1<p<\infty$ we define

$$
\mu_{p}(Q ; F)=\min _{u \in W^{1, p}(Q) \backslash\{0\}}\left\{\frac{\int_{Q}|\nabla u|^{p} \mathrm{~d} x}{\int_{Q}|u|^{p} \mathrm{~d} x}: u=0 \text { on } F\right\} .
$$

We first observe that $F$ is a compact set with positive $(N-1)$-dimensional Hausdorff measure; thus, by [23, Theorem 10.1.2] we can infer the existence of a constant $C=C(N, p, F)>0$ 
such that

$$
\frac{1}{C} \int_{Q}|u|^{p} \mathrm{~d} x \leq \int_{Q}|\nabla u|^{p} \mathrm{~d} x, \quad \text { for every } u \in W^{1, p}(Q) \text { such that } u=0 \text { on } F .
$$

This shows that $\mu_{p}(Q ; F)>0$.

For every $\varepsilon>0$, we consider $u_{\varepsilon} \in C_{0}^{\infty}(E) \backslash\{0\}$ such that

$$
\lambda_{p}^{1}(E)+\varepsilon>\frac{\int_{E}\left|\nabla u_{\varepsilon}\right|^{p} \mathrm{~d} x}{\int_{E}\left|u_{\varepsilon}\right|^{p} \mathrm{~d} x} .
$$

We now observe that for every $z \in \mathbb{Z}^{N}$, there holds

$$
\int_{Q+z}\left|\nabla u_{\varepsilon}\right|^{p} \mathrm{~d} x \geq \mu(Q, F) \int_{Q+z}\left|u_{\varepsilon}\right|^{p} \mathrm{~d} x
$$

thanks to the fact that $u_{\varepsilon}$ vanishes on (the relevant translated copy of) $F$ and to the fact that $\mu_{p}(Q, F)=\mu_{p}(Q+z, F+z)$. By using this information, we get

$$
\begin{aligned}
\int_{E}\left|\nabla u_{\varepsilon}\right|^{p} \mathrm{~d} x & =\sum_{z \in \mathbb{Z}^{N}} \int_{Q+z}\left|\nabla u_{\varepsilon}\right|^{p} \mathrm{~d} x \\
& \geq \mu_{p}(Q, F) \sum_{z \in \mathbb{Z}^{N}} \int_{Q+z}\left|u_{\varepsilon}\right|^{p} \mathrm{~d} x=\mu_{p}(Q, F) \int_{E}\left|u_{\varepsilon}\right|^{p} \mathrm{~d} x .
\end{aligned}
$$

By recalling the choice of $u_{\varepsilon}$, we then get

$$
\lambda_{p}^{1}(E)+\varepsilon \geq \mu_{p}(Q ; F) .
$$

Thanks to the arbitrariness of $\varepsilon>0$ and to the fact that $\widetilde{\Omega}_{n} \subset E$, this finally gives

$$
\lambda_{p}^{1}\left(\widetilde{\Omega}_{n}\right) \geq \lambda_{p}^{1}(E) \geq \mu_{p}(Q ; F), \quad \text { for every } n \in \mathbb{N},
$$

as desired.

Proof of (A.3). We recall that

$$
\widetilde{\Omega}_{n}=\bigcup_{z \in \mathbb{Z}_{n}^{N}}(\Omega+z)=\left[-n-\frac{1}{2}, n+\frac{1}{2}\right]^{N} \backslash \bigcup_{z \in \mathbb{Z}_{n}^{N}}(F+z),
$$

and that each $(N-1)$-dimensional set $F+z$ has null $(s, p)$-capacity, thanks to Remark 5.5. By using Proposition 5.2, we also obtain

$$
\operatorname{cap}_{s, p}\left(\bigcup_{z \in \mathbb{Z}_{n}^{N}}(F+z)\right)=0 .
$$

Then by Proposition 5.3, we get

$$
\lambda_{p}^{s}\left(\widetilde{\Omega}_{n}\right)=\lambda_{p}^{s}\left(\left[-n-\frac{1}{2}, n+\frac{1}{2}\right]^{N}\right)=(2 n+1)^{-s p} \lambda_{p}^{s}(Q) .
$$

This is turn gives the desired conclusion (A.3). 


\section{Appendix B. One-dimensional Hardy inequality}

In the proof of Proposition 4.5, we used the following general form of the one-dimensional Hardy inequality. (The classical case corresponds to $\alpha=p-1$ below.) This can be found, for example, in [23, p. 39]. For the sake of completeness, we give a sketch of a proof based on Picone's inequality. ${ }^{7}$

Lemma B.1 Let $1<p<\infty$ and $\alpha>0$. For every $f \in C_{0}^{\infty}((0, T])$ we have

$$
\left(\frac{\alpha}{p}\right)^{p} \int_{0}^{T} \frac{|f(t)|^{p}}{t^{\alpha}} \frac{\mathrm{d} t}{t} \leq \int_{0}^{T} \frac{\left|f^{\prime}(t)\right|^{p}}{t^{\alpha}} t^{p} \frac{\mathrm{d} t}{t} .
$$

Proof We take $0<\beta<\alpha /(p-1)$ and consider the function $\varphi(t)=t^{\beta}$. Observe that this solves

$$
\begin{aligned}
-\left(\left|\varphi^{\prime}(t)\right|^{p-2} \varphi^{\prime}(t) t^{p-\alpha-1}\right)^{\prime} & =\beta^{p-1}(\alpha-\beta(p-1)) t^{\beta(p-1)-\alpha-1} \\
& =\beta^{p-1}(\alpha-\beta(p-1)) t^{-\alpha-1} \varphi(t)^{p-1} .
\end{aligned}
$$

Thus, for every $\psi \in C_{0}^{\infty}((0, T])$ we have the weak formulation

$$
\beta^{p-1}(\alpha-\beta(p-1)) \int_{0}^{T} \frac{\varphi^{p-1}}{t^{\alpha}} \psi \frac{\mathrm{d} t}{t}=\int_{0}^{T} \frac{\left|\varphi^{\prime}\right|^{p-2} \varphi^{\prime}}{t^{\alpha}} \psi^{\prime} t^{p} \frac{\mathrm{d} t}{t} .
$$

We take $\varepsilon>0$ and $f \in C_{0}^{\infty}((0, T])$ nonnegative, we insert the test function

$$
\psi=\frac{f^{p}}{(\varepsilon+\varphi)^{p-1}},
$$

in the previous integral identity. By using Picone's inequality, we then obtain

$$
\begin{aligned}
\beta^{p-1}(\alpha-\beta(p-1)) \int_{0}^{T} \frac{\varphi^{p-1}}{(\varepsilon+\varphi)^{p-1}} \frac{f^{p}}{t^{\alpha}} \frac{\mathrm{d} t}{t} & =\int_{0}^{T} \frac{\left|\varphi^{\prime}\right|^{p-2} \varphi^{\prime}}{t^{\alpha}}\left(\frac{f^{p}}{(\varepsilon+\varphi)^{p-1}}\right)^{\prime} t^{p} \frac{\mathrm{d} t}{t} \\
& \leq \int_{0}^{T} \frac{\left|f^{\prime}(t)\right|^{p}}{t^{\alpha}} t^{p} \frac{\mathrm{d} t}{t} .
\end{aligned}
$$

If we take the limit as $\varepsilon$ goes to 0 , by Fatou's lemma we get

$$
\beta^{p-1}(\alpha-\beta(p-1)) \int_{0}^{T} \frac{f^{p}}{t^{\alpha}} \frac{\mathrm{d} t}{t} \leq \int_{0}^{T} \frac{\left|f^{\prime}(t)\right|^{p}}{t^{\alpha}} t^{p} \frac{\mathrm{d} t}{t} .
$$

The previous inequality holds true for every $0<\beta<\alpha /(p-1)$ and $\beta^{p-1}(\alpha-\beta(p-1))$ is maximal for $\beta=\alpha / p$. This concludes the proof.

\section{Appendix C. A geometric lemma}

When comparing the norms of $\mathcal{X}_{0}^{s, p}(\Omega)$ and $\mathcal{D}_{0}^{s, p}(\Omega)$ for a convex set, we used the following geometric result. We recall that

7 For $u, v$ differentiable functions with $v \geq 0$ and $u>0$, we have the pointwise inequality

$$
\left|u^{\prime}\right|^{p-2} u^{\prime}\left(\frac{v^{p}}{u^{p-1}}\right)^{\prime} \leq\left|v^{\prime}\right|^{p} .
$$


Fig. 2 The case (ii) in the proof of Lemma C.1. Colored in red, the distance of $t z$ from $\partial C_{z}$ (color figure online)

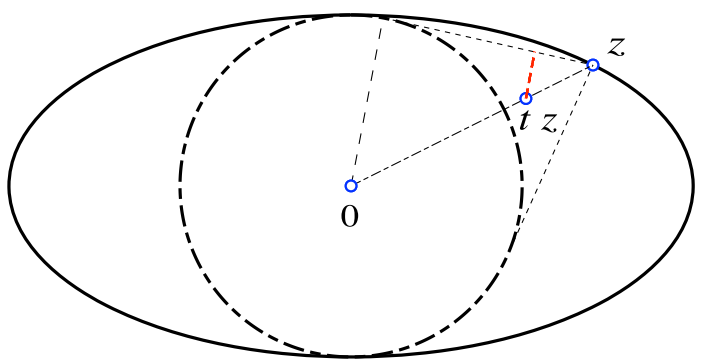

$$
R_{\Omega}=\sup _{x \in \Omega} \operatorname{dist}(x, \partial \Omega),
$$

is the inradius of $\Omega$, i.e., the radius of the largest ball inscribed in $\Omega$.

Lemma C.1 Let $\Omega \subset \mathbb{R}^{N}$ be an open convex set such that $R_{\Omega}<+\infty$. Let $x_{0} \in \Omega$ be a point such that

$$
\operatorname{dist}\left(x_{0}, \partial \Omega\right)=R_{\Omega} \text {. }
$$

Then for every $0<t<1$ we have

$$
\operatorname{dist}\left(x_{0}+t\left(\Omega-x_{0}\right), \partial \Omega\right) \geq(1-t) R_{\Omega} .
$$

Proof Without the loss of generality, we can assume that $0 \in \Omega$ and that $x_{0}=0$. Clearly, it is sufficient to prove that

$$
\operatorname{dist}(\partial(t \Omega), \partial \Omega) \geq(1-t) R_{\Omega} .
$$

Every point of $\partial(t \Omega)$ is of the form $t z$, with $z \in \partial \Omega$. We now take the cone $C_{z}$, obtained as the convex envelope of $B_{R_{\Omega}}(0)$ and the point $z$. By convexity of $\Omega$, we have of course $C_{z} \subset \Omega$. We thus obtain

$$
\operatorname{dist}(t z, \partial \Omega) \geq \operatorname{dist}\left(t z, \partial C_{z}\right) .
$$

We now distinguish two cases:

(i) $|z|=R_{\Omega}$;

(ii) $|z|>R_{\Omega}$.

When alternative (i) occurs, then $C_{z}=B_{R_{\Omega}}(0)$ and thus

$$
\operatorname{dist}\left(t z, \partial C_{z}\right)=\operatorname{dist}\left(t z, \partial B_{R_{\Omega}}(0)\right)=|t z-z|=(1-t)|z|=(1-t) R_{\Omega} .
$$

By using this in (C.1), we get the desired estimate.

If on the contrary we are in case (ii), then by elementary geometric considerations we have

$$
\frac{\operatorname{dist}\left(t z, \partial C_{z}\right)}{|t z-z|}=\frac{R_{\Omega}}{|z|}
$$

see Fig. 2. This gives again the desired conclusion. 


\section{References}

1. Adams, R.A.: Sobolev Spaces. Pure and Applied Mathematics, vol. 65. Academic Press, New York (1975)

2. Adams, R.A., Fournier, J.J.F.: Sobolev Spaces. Pure and Applied Mathematics (Amsterdam), vol. 140, 2nd edn. Elsevier/Academic Press, Amsterdam (2003)

3. Bennett, C., Sharpley, R.: Interpolation of Operators. Pure and Applied Mathematics, vol. 129. Academic Press, Boston (1988)

4. Bergh, J., Löfström, J.: Interpolation Spaces. An Introduction. Grundlehren der Mathematischen Wissenschaften, vol. 223. Springer, Berlin (1976)

5. Bourgain, J., Brezis, H., Mironescu, P.: Another Look at Sobolev Spaces. Optimal Control and Partial Differential Equations, pp. 439-455. IOS, Amsterdam (2001)

6. Bramble, J.H.: Interpolation between Sobolev spaces in Lipschitz domains with an application to multigrid theory. Math. Comp. 64, 1359-1365 (1995)

7. Brasco, L., Lindgren, E., Parini, E.: The fractional Cheeger problem. Interfaces Free Bound. 16, 419-458 (2014)

8. Brasco, L., Cinti, E.: On fractional Hardy inequalities in convex sets. Discrete Contin. Dyn. Syst. 38, 4019-4040 (2018)

9. Brasco, L., Santambrogio, F.: A sharp estimate à la Calderón-Zygmund for the p-Laplacian. Commun. Contemp. Math. 20, 1750030, 24 (2018)

10. Bucur, C., Valdinoci, E.: Nonlocal Diffusion and Applications. Lecture Notes of the Unione Matematica Italiana, vol. 20. Springer, Unione Matematica Italiana, Cham, Bologna (2016)

11. Chandler-Wilde, S.N., Hewett, D.P., Moiola, A.: Interpolation of Hilbert and Sobolev spaces: quantitative estimates and counterexamples. Mathematika 61, 414-443 (2015)

12. Chen, Z.-Q., Song, R.: Two-sided eigenvalue estimates for subordinate processes in domains. J. Funct. Anal. 226, 90-113 (2005)

13. Deny, J., Lions, J.-L.: Les espaces du type de Beppo Levi. Ann. Inst. Fourier 5, 305-370 (1954)

14. Di Nezza, E., Palatucci, G., Valdinoci, E.: Hitchhiker's guide to the fractional Sobolev spaces. Bull. Sci. Math. 136, 521-573 (2012)

15. Dyda, B.: A fractional order Hardy inequality. Ill. J. Math. 48, 575-588 (2004)

16. Dyda, B., Vähäkangas, A.V.: Characterizations for fractional Hardy inequality. Adv. Calc. Var. 8, 173-182 (2015)

17. Evans, L.C., Gariepy, R.: Measure Theory and Fine Properties of Functions. Studies in Advanced Mathematics. CRC Press, Boca Raton (1992)

18. Franzina, G.: Non-local torsion functions and embeddings. Appl. Analyis (2018). https://doi.org/10.1080/ 00036811.2018 .1463521

19. Gigli, N., Mosconi, S.: The abstract Lewy-Stampacchia inequality and applications. J. Math. Pures Appl. 104, 258-275 (2015)

20. Grisvard, P.: Elliptic Problems in Nonsmooth Domains. Monographs and Studies in Mathematics, vol. 24. Pitman (Advanced Publishing Program), Boston (1985)

21. Jones, P.W.: Quasiconformal mappings and extendability of functions in Sobolev spaces. Acta Math. 147, 71-88 (1981)

22. Lions, J.-L., Magenes, E.: Non-homogeneous Boundary Value Problems and Applications, vol. I. Translated from the French by P. Kenneth. Die Grundlehren der mathematischen Wissenschaften, Band, vol. 181. Springer, New York (1972)

23. Maz'ja, V.G.: Sobolev Spaces. Translated from the Russian by T. O. Shaposhnikova. Springer Series in Soviet Mathematics. Springer, Berlin (1985)

24. Maz'ya, V., Shaposhnikova, T.: On the Bourgain, Brezis, and Mironescu theorem concerning limiting embeddings of fractional Sobolev spaces. J. Funct. Anal. 195, 230-238 (2002)

25. Nikol'skiǔ, S.M.: Approximation of Functions of Several Variables and Imbedding Theorems. Translated from the Russian by John M. Danskin, Jr. Die Grundlehren der Mathematischen Wissenschaften, Band, vol. 205. Springer, New York (1975)

26. Ponce, A.: A new approach to Sobolev spaces and connections to $\Gamma$-convergence. Calc. Var. Partial Differ. Equ. 19, 229-255 (2004)

27. Stein, E.: Singular Integrals and Differentiability Properties of Functions. Princeton Mathematical Series, vol. 30. Princeton University Press, Princeton (1970)

28. Tartar, L.: An Introduction to Sobolev Spaces and Interpolation Spaces. Lecture Notes of the Unione Matematica Italiana, vol. 3. Springer, UMI, Berlin, Bologna (2007)

29. Triebel, H.: Interpolation Theory, Function Spaces, Differential Operators. North-Holland, Amsterdam (1978) 
30. Triebel, H.: Theory of Function Spaces. III. Monographs in Mathematics, vol. 100. Birkhäuser Verlag, Basel (2006)

31. Triebel, H.: Theory of Function Spaces. II. Monographs in Mathematics, vol. 84. Birkhäuser Verlag, Basel (1992)

32. Triebel, H.: Theory of Function Spaces. Monographs in Mathematics, vol. 78. Birkhäuser Verlag, Basel (1983)

Publisher's Note Springer Nature remains neutral with regard to jurisdictional claims in published maps and institutional affiliations. 